\title{
POLY-POISSON STRUCTURES
}

\author{
DAVID IGLESIAS, JUAN CARLOS MARRERO, AND MIGUEL VAQUERO
}

\begin{abstract}
In this paper we introduce poly-Poisson structures as a higher-order extension of Poisson structures. It is shown that any poly-Poisson structure is endowed with a polysymplectic foliation. It is also proved that if a Lie group acts polysymplectically on a polysymplectic manifold then, under certain regularity conditions, the reduced space is a poly-Poisson manifold. In addition, some interesting examples of poly-Poisson manifolds are discussed.
\end{abstract}

\section{Contents}

1. Introduction

2. Polysymplectic structures

3. K-poly-Poissson structures

4. Poly-Poisson structures and reduction of polysymplectic structures 16

Appendix: Lie algebroids and fiberwise linear Poisson structures 21

References

\section{INTRODUCTION}

As it is well known, symplectic manifolds play a fundamental role in the Hamiltonian formulation of classical mechanics. Indeed, given a configuration space $Q$, its cotangent bundle $T^{*} Q$, the phase space, is endowed with a symplectic structure and the Hamilton's equations associated to a Hamiltonian $H: T^{*} Q \rightarrow \mathbb{R}$ can be interpreted as the flow of the Hamiltonian vector field associated with $H$.

Under the existence of symmetries, it is possible to perform a reduction procedure in which some of the variables are reduced. The interest of the reduction procedure is twofold: not only we are able to reduce the dynamics but it is a way to generate new examples of symplectic manifolds. One of the reduction methods is the Marsden-Weinstein-Meyer reduction procedure [16]: Given a Hamiltonian action of a Lie group $G$ on a symplectic manifold $(M, \Omega)$ with equivariant moment map $J: M \rightarrow \mathfrak{g}^{*}$, it is possible to obtain a symplectic structure on the quotient manifold $J^{-1}(\mu) / G_{\mu}$. An interesting example is the action of a Lie group $G$ on its cotangent bundle $T^{*} G$ by cotangent lifts of left translations and the moment map $J: T^{*} G \rightarrow \mathfrak{g}^{*}$ is given by $J\left(\alpha_{g}\right)=\left(T_{e} R_{g}\right)^{*}\left(\alpha_{g}\right)$. Here, the reduced space $J^{-1}(\mu) / G_{\mu}$ is just the coadjoint orbit along the element $\mu \in \mathfrak{g}^{*}$ endowed with the Kirillov-Kostant-Souriau symplectic structure.

On the other hand, the existence of a Lie group of symmetries for a symplectic manifold is one of the justifications for the introduction of Poisson manifolds, which generalize symplectic manifolds. Indeed if $(M, \Omega)$ is a symplectic manifold and $G$ is a Lie group acting freely and

2010 Mathematics Subject Classification. 53D05, 53D17, 70645.

Key words and phrases. polysymplectic manifolds, poly-Poisson structures, polysymplectic foliation, reduction, Lie algebroids, linear Poisson structures. 
properly on $M$ and preserving the symplectic structure then the quotient manifold $M / G$ is endowed with a Poisson structure in such a way that the projection $\pi: M \rightarrow M / G$ is a Poisson epimorphism. A particular example is the so-called Poisson-Sternberg structure on $T^{*} Q / G$, which is obtained reducing the symplectic structure on $T^{*} Q$ after lifting a free and proper action of a Lie group $G$ on $Q$ to $T^{*} Q$ (see [17] for applications in Mechanics). Any Poisson structure induces a foliation with symplectic leaves, the symplectic foliation. For symplectic manifolds there is only one leaf: the manifold itself. The other interesting example is the Lie Poisson structure on $\mathfrak{g}^{*}$, the dual space of any Lie algebra $\mathfrak{g}$. In this case, the symplectic leaves are the coadjoint orbits previously obtained as reduced symplectic spaces.

In 10, polysymplectic manifolds were introduced as a natural vector-valued extension of symplectic manifolds. A polysymplectic structure on a manifold $M$ is a closed nondegenerate $\mathbb{R}^{k}$-valued 2-form on $M$. The physical motivation was obtain a geometric framework in which develop field theories for Lagrangians defined on the first-jet bundle of a trivial fibration $p: M=$ $N \times U \rightarrow U, U \subseteq \mathbb{R}^{k}$ being the parameter space of the theory. In this case, the natural polysymplectic manifold is $L Q$, the tangent frame bundle of $Q$. More generally, it is possible to consider the cotangent bundle of $k$-covelocities $\left(T_{k}^{1}\right)^{*} Q:=T^{*} Q \oplus . \stackrel{(k}{.} \oplus T^{*} Q$.

In addition, in [10] the author considers polysymplectic actions of Lie groups on polysymplectic manifolds and the notion of a polysymplectic moment map $J: M \rightarrow \mathfrak{g}^{*} \otimes \mathbb{R}^{k}$ is introduced. We note that other moment map theories with different target spaces (such as Lie groups or homogeneous spaces [2, 3, 4) have been proposed but this one fits in a different framework. In addition, in this situation, it is developed a reduction procedure mimicking the symplectic case and, under certain conditions, a polysymplectic structure on $J^{-1}(\mu) / G_{\mu}$ is obtained. We must remark here that, the assumed conditions in [10] do not assure that the quotient space $J^{-1}(\mu) / G_{\mu}$ is polysymplectic because this fact is based on a wrong result (see Lemma 7.5 in [10]). In [15], a right version of the Marsden-Weinstein reduction theorem in the polysymplectic setting is proved.

As an example of the reduction procedure, a polysymplectic structure on $k$-coadjoint orbits $\mathcal{O}_{\left(\mu_{1}, \ldots, \mu_{k}\right)}$, for $\left(\mu_{1}, \ldots, \mu_{k}\right) \in \mathfrak{g}^{*} \otimes \mathbb{R}^{k}=\mathfrak{g}^{*} \times \stackrel{(k}{ } \times \mathfrak{g}^{*}$ is obtained. A natural question arises: Is there a geometric structure on $\mathfrak{g}^{*} \times \stackrel{(k}{.} \times \mathfrak{g}^{*}$ which admits a polysymplectic foliation whose leaves are the $k$-coadjoint orbits?

In this paper, it is proposed an answer to this question introducing the notion of a polyPoisson structure as a generalization of Poisson structures as well as of polysymplectic structures. Contrary to what would be expected, these are not just $\mathbb{R}^{k}$-valued 2 -vector fields. Indeed, if one starts from a polysymplectic structure $\bar{\omega}$, one can construct a vector-bundle morphism $\bar{\omega}^{b}: T M \rightarrow\left(T_{k}^{1}\right)^{*} M$ and the non-degeneracy of $\bar{\omega}$ is equivalent to $\bar{\omega}^{b}$ being injective (see Proposition 2.3). However, if $k>2$ this does not imply that $\bar{\omega}^{b}$ is an isomorphism, so it does not make sense to take the inverse of this map. Our approach is the following one: if $S:=\operatorname{Im}\left(\bar{\omega}^{b}\right)$ then one can consider the inverse of $\bar{\omega}^{b}$ restricted to $S$, that is,

$$
\bar{\Lambda}^{\sharp}:=\left(\bar{\omega}^{b}\right)_{\mid S}^{-1}: S \rightarrow T M .
$$

Thus, this fact suggests to introduce the notion of a poly-Poisson structure as a pair $\left(S, \bar{\Lambda}^{\sharp}\right)$, where $S$ is a vector subbundle of $\left(T_{k}^{1}\right)^{*} M$ and $\bar{\Lambda}^{\sharp}: S \rightarrow T M$ is a vector bundle morphism satisfying several conditions (see Definition 13.1). As for Poisson manifolds, any poly-Poisson manifold is endowed with a foliation, whose associated distribution is $\bar{\Lambda}^{\sharp}(S)$, with polysymplectic leaves. Moreover, a second motivation from Poisson geometry is the following one. If $G$ is a Lie group which acts polysymplectically on a polysymplectic manifold then it is possible to obtain, under some mild regularity conditions, a poly-Poisson structure on the reduced space $M / G$.

The paper is organized as follows. In section 2, it is recalled the notion of a polysymplectic structure and it is obtained a characterization in terms of bundle maps. After giving some examples, in section 3 we introduce the notion of a poly-Poisson structure. After that it is 
shown that any poly-Poisson manifold has associated a generalized foliation and that on each leaf it is induced a polysymplectic structure. In order to obtain examples, it is proposed a method to construct poly-Poisson structures out of a family of Poisson structures. As an application, given a linear Poisson structure on a vector bundle $E^{*}$ with base space $Q$, it is described a polyPoisson structure on the Whitney sum $E^{*} \oplus \ldots \oplus E^{*}$ and, in particular, on the frame bundle $L E \rightarrow Q$. In section 5, it is developed a reduction procedure for polysymplectic manifolds. More precisely, if $G$ is a Lie group acting polysymplectically on a polysymplectic manifold $(M, \Omega)$ then, under certain regularity conditions, the quotient manifold $M / G$ is endowed with a poly-Poisson structure. As an application, given a principal $G$-bundle $Q \rightarrow Q / G$ with total space $Q$ it is possible to obtain a poly-Poisson structure on the Withney sum $T^{*} Q / G \oplus \stackrel{(k}{*} \oplus T^{*} Q / G$ as the reduction by the Lie group $G$ of the cotangent bundle of $k$-covelocities $T^{*} Q \oplus . \stackrel{(k}{{ }^{*}} \oplus T^{*} Q$. As a consequence, we deduce that the reduction of the tangent frame bundle $L Q \rightarrow Q$ is the frame bundle of the Atiyah algebroid $T Q / G \rightarrow Q / G$. The paper ends with and appendix which contains some basic definitions and results on Lie algebroids and fiberwise linear Poisson structures on vector bundles.

We finally remark that there is a relation with Dirac structures (see [9]). Dirac structures provide a common framework to Poisson and presymplectic manifolds. These are integrable Lagrangian subbundles of $T M \oplus T^{*} M$. To interpret poly-Poisson structures in this setting, we could consider the bundle $T M \oplus\left(T_{k}^{1}\right)^{*} M$, which is endowed with a nondegenerate symmetric bilinear form with values in $\mathbb{R}^{k}[12$. We postpone to a future work the details of this, hoping it will provide a framework to analyze reduction (see, for instance, [7, 8]).

Notation: If $Q$ is a differentiable manifold of dimension $n,\left(T_{k}^{1}\right)^{*} Q$ will denote the cotangent bundle of $k$-covelocities of $Q$, that is, the Whitney sum of $k$-copies of the cotangent bundle,

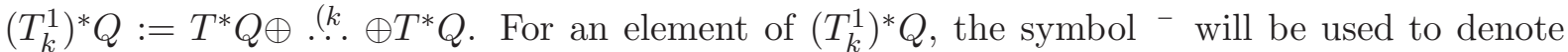
it, and subscripts without ${ }^{-}$to denote its components, i.e. $\bar{\alpha} \in\left(T_{k}^{1}\right)^{*} Q$ and $\bar{\alpha}=\left(\alpha_{1}, \ldots, \alpha_{k}\right)$ where $\alpha_{i} \in T^{*} Q$ for $i \in\{1, \ldots, k\}$. The same criterion will be used for 2 -forms and morphisms. If $\bar{\alpha} \in\left(T_{k}^{1}\right)_{p}^{*} Q$ are $k 1$-forms over the same point, $X \in T_{p} Q$ is a tangent vector and $W \subset T_{p} Q$ is a vector subspace, we write $\bar{\alpha}(X)=\left(\alpha_{1}(X), \ldots, \alpha_{k}(X)\right) \in \mathbb{R}^{k}$ for the evaluation and the restriction $\bar{\alpha}_{\mid W}=\left(\alpha_{1 \mid W}, \ldots, \alpha_{k \mid W}\right) \in W^{*} \oplus \ldots \oplus W^{*}$ is just the restriction of every component. If $\bar{\alpha}$ is a section of $\left(T_{k}^{1}\right)^{*} Q$ and $X$ a vector field we will write $\mathcal{L}_{X} \bar{\alpha}=\left(\mathcal{L}_{X} \alpha_{1}, \ldots, \mathcal{L}_{X} \alpha_{k}\right)$ and if $\bar{f}$ is a $\mathbb{R}^{k}$-valued function, $\bar{f}=\left(f_{1}, \ldots, f_{k}\right)$, then we will write $\mathrm{d} \bar{f}=\left(\mathrm{d} f_{1}, \ldots, \mathrm{d} f_{k}\right)$. When we deal with the fibers of bundles over a manifold, we sometimes omit reference to base point when it is clear or when what are we saying is true for all points of the base manifold.

\section{Polysymplectic structures}

In this section, it is recalled the concept of a polysymplectic structure, which is one of the possible higher order analogues of a symplectic structure (see [10]).

Definition 2.1. A $k$-polysymplectic structure on a manifold $M$ is a closed nondegenerate $\mathbb{R}^{k}$ valued 2 -form

$$
\bar{\omega}=\sum_{A=1}^{k} \omega^{A} \otimes e_{A}
$$

where $\left\{e_{1}, \ldots, e_{k}\right\}$ denotes the canonical basis of $\mathbb{R}^{k}$.

Equivalently, $\bar{\omega}$ can be seen as a family of $k$ closed 2 -forms $\left(\omega^{1}, \ldots, \omega^{k}\right)$ such that

$$
\cap_{A=1}^{k} \operatorname{Ker} \omega^{A}=0 \text {. }
$$

The pair $(M, \bar{\omega})=\left(M, \omega^{1}, \ldots, \omega^{k}\right)$ is called a $k$-polysymplectic manifold or simply a polysymplectic manifold. 
Remark 2.2. It is clear that the notion of a 1-polysymplectic structure coincides with the definition of a symplectic structure.

Polysymplectic structures can be thought of as vector bundle morphisms, obtaining an equivalent definition of polysymplectic manifolds.

Proposition 2.3. A $k$-polysymplectic structure on a manifold $M$ is a vector bundle morphism $\bar{\omega}^{b}: T M \rightarrow\left(T_{k}^{1}\right)^{*} M$ of the tangent bundle $T M$ to $M$ on the cotangent bundle of $k$-covelocities of $M$ satisfying the conditions:

i) Skew-symmetry:

$$
\bar{\omega}^{b}(X)(X)=(0, \ldots, 0), \quad X \in T_{p} M, p \in M .
$$

ii) Non degeneracy ( $\bar{\omega}^{b}$ is a monomorphism):

$$
\operatorname{Ker}\left(\bar{\omega}^{b}\right)=\{0\} .
$$

iii) Integrability condition:

$$
\bar{\omega}^{b}([X, Y])=\mathcal{L}_{X} \bar{\omega}^{b}(Y)-\mathcal{L}_{Y} \bar{\omega}^{b}(X)+\mathrm{d}\left(\bar{\omega}^{b}(X)(Y)\right), \quad X, Y \in \mathfrak{X}(M) .
$$

Proof. Let $\bar{\omega}^{b}: T M \rightarrow\left(T_{k}^{1}\right)^{*} M$ be a vector bundle morphism (over the identity of $M$ ). Then, condition i) is equivalent to the existence of $k 2$-forms $\omega^{1}, \ldots, \omega^{k}$ on $M$ such that

$$
\bar{\omega}^{b}(X)=\left(\left(\omega^{1}\right)^{b}(X), \ldots,\left(\omega^{k}\right)^{b}(X)\right), \quad \text { for } X \in T M .
$$

On the other hand, ii) holds if and only if

$$
\cap_{A=1}^{k} \operatorname{Ker} \omega^{A}=\{0\} .
$$

Finally, using the following relation

$$
i_{Y} i_{X} \mathrm{~d} \Phi+\Phi^{b}[X, Y]=\mathcal{L}_{X} \Phi^{b}(Y)-\mathcal{L}_{Y} \Phi^{b}(X)+\mathrm{d}\left(\Phi^{b}(X)(Y)\right), \quad \text { for } X, Y \in \mathfrak{X}(M),
$$

which is valid for any arbitrary 2 -form $\Phi$ on $M$, we deduce that (2.2) holds if and only if $\omega^{A}$ is a closed 2-form, for all $A$. This proves the result.

Next, an interesting example of a polysymplectic manifold will be presented.

Example 2.4 (The tangent frame bundle [18]). Let $L M$ be the tangent frame bundle of $M$, that is, the elements of $L M$ are pairs $\left(m,\left\{e_{i}\right\}\right)$, where $\left\{e_{i}\right\}, i=1, \ldots n$, is a linear frame at $m \in M$. There is a right action of $G L(n), n=\operatorname{dim} M$, on $L M$ by

$$
\left(m, e_{i}\right) \cdot g=\left(m, e_{i} g_{i j}\right), \quad \text { for }\left(m, e_{i}\right) \in L M, g=\left(g_{i j}\right) \in G L(n) .
$$

With this action $L M \rightarrow M$ is a principal $G L(n)$-bundle.

On $L M$ there exists a canonical vector-valued 1-form $\vartheta \in \Omega^{1}\left(L M, \mathbb{R}^{n}\right)$, called the soldering one-form, which is defined by

$$
\begin{aligned}
\vartheta(u): \quad T_{u}(L M) & \rightarrow \mathbb{R}^{n} \\
X & \mapsto \vartheta(u)(X)=u^{-1}\left(T_{u} \pi(X)\right),
\end{aligned}
$$

where $\pi: L M \rightarrow M$ is the canonical projection and $u=\left(m, e_{i}\right) \in L M$ is seen as the non-singular linear map $u: \mathbb{R}^{n} \rightarrow T_{m} M,\left(v^{1}, \ldots, v^{n}\right) \mapsto \sum_{i=1}^{n} v^{i} e_{i}$.

The soldering one-form endowes $L M$ with a $n$-polysymplectic structure given by

$$
\omega=-d \vartheta \text {. }
$$

(for more details, see [18]). 
Next, it will be shown a method to construct a polysymplectic structure out of a family of symplectic manifolds. Indeed, this construction is the model for a regular polysymplectic manifold.

Example 2.5. Let $\left(M_{A}, \Omega^{A}\right)$ be a symplectic manifold, with $A \in\{1, \ldots, k\}$, and $\pi_{A}: M \rightarrow M_{A}$ a fibration. Denote by $\omega^{A}$ the presymplectic form on $M$ given by

$$
\omega^{A}=\pi_{A}^{*}\left(\Omega^{A}\right), \text { for } A \in\{1, \ldots, k\} .
$$

It is easy to prove that $\omega^{A}$ has constant rank. In fact,

$$
\operatorname{Ker} \omega^{A}=\operatorname{Ker}\left(T \pi_{A}\right) \text {, }
$$

where $T \pi_{A}: T M \rightarrow T M_{A}$ is the tangent map to $\pi_{A}: M \rightarrow M_{A}$. Thus, we deduce that the family of 2-forms $\left(\omega^{1}, \ldots, \omega^{k}\right)$ on $M$ define a $k$-polysymplectic structure if $\cap_{A=1}^{k} \operatorname{Ker}\left(T \pi_{A}\right)=\{0\}$.

Conversely, if $\left(\omega^{1}, \ldots, \omega^{k}\right)$ is a $k$-polysymplectic structure on a manifold $M$ and the 2 -forms $\omega^{A}$ have constant rank then the distributions $\mathcal{F}_{A}$, with $A \in\{1, \ldots, k\}$, given by

$$
x \in M \rightarrow \mathcal{F}_{A}(x)=\operatorname{Ker}\left(\omega^{A}(x)\right) \subseteq T_{x} M
$$

are completely integrable (this follows using that $\omega^{A}$ is closed). In addition, if the space of leaves $M_{A}=M / \mathcal{F}_{A}$ of the foliation $\mathcal{F}_{A}$ is a quotient manifold, for $A \in\{1, \ldots, k\}$, and $\pi_{A}: M \rightarrow$ $M_{A}=M / \mathcal{F}_{A}$ is the canonical projection, we have that the 2 -form $\omega^{A}$ is basic with respect to $\pi_{A}$. Therefore, there exists a unique 2 -form $\Omega^{A}$ on $M_{A}$ satisfying

$$
\omega^{A}=\pi_{A}^{*}\left(\Omega^{A}\right) .
$$

Finally, since the map $\pi_{A}^{*}: \Omega^{p}\left(M_{A}\right) \rightarrow \Omega^{p}(M)$ is a monomorphism of modules, we deduce that $\Omega^{A}$ is a symplectic form on $M_{A}$.

Some particular examples of the previous general construction are the following ones.

Example 2.6 (The product of symplectic manifolds). Let $\left(M_{A}, \Omega^{A}\right)$ be a symplectic manifold, with $A \in\{1, \ldots, k\}$, and $M=M_{1} \times \ldots \times M_{k}$. Then, it is clear that $M$ admits a $k$-polysymplectic structure $\left(\omega^{1}, \ldots, \omega^{k}\right)$. It is sufficient to take

$$
\omega^{A}=\operatorname{pr}_{A}^{*}\left(\Omega^{A}\right), \quad \text { for } A \in\{1, \ldots, k\},
$$

where $\operatorname{pr}_{A}: M \rightarrow M_{A}$ is the canonical projection from $M$ on $M_{A}$.

Example 2.7 (The cotangent bundle of $k$-covelocities of a manifold). It is well known that, given an arbitrary manifold $Q$, its cotangent bundle $T^{*} Q$ is endowed with a canonical symplectic structure in the following way. If $\theta$ is the Liouville 1 -form on $T^{*} Q$ then $\omega=-\mathrm{d} \theta$ is a symplectic 2 -form on $T^{*} Q$ (see, for instance, [1, 13]).

Next, using this symplectic structure and the previous general construction, we will obtain a $k$-polysymplectic structure on the cotangent bundle of $k$-covelocities $\left(T_{k}^{1}\right)^{*} Q:=T^{*} Q \oplus . \stackrel{(k}{*} \oplus T^{*} Q$ of $Q$. Denote by $\pi_{Q}^{k}:\left(T_{k}^{1}\right)^{*} Q \rightarrow Q$ the canonical projection and by $\pi_{Q}^{k, A}:\left(T_{k}^{1}\right)^{*} Q \rightarrow T^{*} Q$ the fibration defined by

$$
\pi_{Q}^{k, A}\left(\alpha_{1}, \ldots, \alpha_{k}\right)=\alpha_{A}, \quad \text { for } A \in\{1, \ldots k\} .
$$

If $\left(q^{i}\right)$ are local coordinates on $U \subseteq Q$, then the induced local coordinates $\left(q^{i}, p_{i}^{A}\right)$ on $\left(\pi_{Q}^{k}\right)^{-1}(U)$ are given by

$$
q^{i}\left(\alpha_{1}^{q}, \ldots, \alpha_{k}^{q}\right)=q^{i}(q), \quad p_{i}^{A}\left(\alpha_{1}^{q}, \ldots, \alpha_{k}^{q}\right)=\alpha_{A}^{q}\left(\frac{\partial}{\partial q^{i}}{ }_{\mid q}\right), \quad 1 \leq i \leq n ; 1 \leq A \leq k .
$$

Moreover, the local expression of $\pi_{Q}^{k, A}$ is

Then, it is clear that

$$
\pi_{Q}^{k, A}\left(q^{i}, p_{i}^{1}, \ldots, p_{i}^{k}\right)=\left(q^{i}, p_{i}^{A}\right) .
$$

$$
\cap_{A=1}^{k} \operatorname{Ker}\left(T \pi_{Q}^{k, A}\right)=\{0\} .
$$


Therefore, $\left(\omega^{1}, \ldots, \omega^{k}\right)$ is a $k$-polysymplectic structure on $\left(T_{k}^{1}\right)^{*} Q$, where $\omega^{A}$ is the 2 -form on $\left(T_{k}^{1}\right)^{*} Q$ defined by

$$
\omega^{A}=\left(\pi_{Q}^{k, A}\right)^{*} \omega .
$$

Note that the local expression of $\omega^{A}$ is

$$
\omega^{A}=d q^{i} \wedge d p_{i}^{A} .
$$

Remark 2.8. Let $Q$ be a manifold of dimension $n$. Then, from Example 2.4, the tangent frame bundle $L Q$ is endowed with a polysymplectic structure $-\mathrm{d} \vartheta$. On the other hand, we have just seen that the cotangent bundle of $n$-covelocities, $\left(T_{n}^{1}\right)^{*} Q$, also is a polysymplectic manifold.

In addition, $L Q$ is an open subset of $\left(T_{n}^{1}\right)^{*} Q$, where the inclusion $\iota: L Q \rightarrow\left(T_{n}^{1}\right)^{*} Q$ is given by $u \mapsto\left(\operatorname{pr}_{1} \circ u^{-1}, \ldots, \operatorname{pr}_{n} \circ u^{-1}\right)$. Note that, $\pi=\pi_{Q}^{n} \circ \iota$, where $\pi: L Q \rightarrow Q$ and $\pi_{Q}^{n}:\left(T_{n}^{1}\right)^{*} Q \rightarrow Q$ are the projections. Let us see that the restriction of the poly-symplectic structure on $\left(T_{n}^{1}\right)^{*} Q$ is just $\mathrm{d} \vartheta$. More precisely, let us show that

$$
\iota^{*}\left(\sum_{A=1}^{n}\left(\pi_{Q}^{n, A}\right)^{*}(\theta) \otimes e_{A}\right)=\vartheta,
$$

where $\left\{e_{1}, \ldots, e_{n}\right\}$ denotes the canonical basis of $\mathbb{R}^{n}, \theta$ is the Liouville 1 -form, $\pi_{Q}^{n, A}:\left(T_{n}^{1}\right)^{*} Q \rightarrow$ $T^{*} Q$ is the projection on the $A$-factor, for $A \in\{1, \ldots n\}$, and $\vartheta$ is the soldering 1 -form. If $\left(X_{1}, \ldots, X_{n}\right) \in T\left(\left(T_{n}^{1}\right)^{*} Q\right)$ then

$$
\begin{aligned}
& \left(\iota^{*}\left(\sum_{A=1}^{k}\left(\pi_{Q}^{n, A}\right)^{*}(\theta) \otimes e_{A}\right)(u)\right)\left(X_{1}, \ldots, X_{n}\right)= \\
& \quad=\left(\sum_{A=1}^{k}\left(\pi_{Q}^{n, A}\right)^{*}(\theta) \otimes e_{A}\right)\left(\operatorname{pr}_{1} \circ u^{-1}, \ldots, \operatorname{pr}_{n} \circ u^{-1}\right)\left(X_{1}, \ldots, X_{n}\right) \\
& =\quad\left(\operatorname{pr}_{1} \circ u^{-1}\left(T \pi_{Q}\left(X_{1}\right)\right), \ldots, \operatorname{pr}_{n} \circ u^{-1}\left(T \pi_{Q}\left(X_{n}\right)\right)\right)=u^{-1}\left(T \pi\left(X_{1}, \ldots, X_{n}\right)\right) \\
& \quad=\vartheta(u)\left(X_{1}, \ldots, X_{n}\right) .
\end{aligned}
$$

Example 2.9 ( $k$-coadjoint orbits [10, 15]). In this example, it is described a $k$-polysymplectic structure on every $k$-coadjoint orbit associated with a Lie group $G$. In the particular case when $k=1$, it is recovered the standard symplectic structure on the coadjoint orbits of $G$.

We will recall the definition of such a structure. Let $G$ be a Lie group and $\mathfrak{g}$ be its Lie algebra. Consider the coadjoint action

$$
\text { Coad: } \begin{aligned}
G \times \mathfrak{g}^{*} & \rightarrow \mathfrak{g}^{*} \\
(g, \mu) & \mapsto \operatorname{Coad}(g, \mu)=\mu \circ A d_{g^{-1}} .
\end{aligned}
$$

The orbit of $\mu \in \mathfrak{g}^{*}$ in $\mathfrak{g}^{*}$ under this action, denoted by

$$
\mathcal{O}_{\mu}=\{\operatorname{Coad}(g, \mu) \mid g \in G\},
$$

is equipped with a symplectic structure $\omega_{\mu}$ defined by

$$
\omega_{\mu}(\nu)\left(\xi_{\mathfrak{g}^{*}}(\nu), \eta_{\mathfrak{g}^{*}}(\nu)\right)=-\nu[\xi, \eta]
$$

where $\nu$ is an arbitrary point of $\mathcal{O}_{\mu}, \xi, \eta \in \mathfrak{g}$ and $\xi_{\mathfrak{g}^{*}}(\nu), \eta_{\mathfrak{g}^{*}}(\nu)$ are the infinitesimal generators of the coadjoint action associated with $\xi$ and $\eta$ at the point $\nu$ (for more details see, for instance, [1. p. 303]).

The extension to the polysymplectic setting is as follows. Define an action of $G$ over $\mathfrak{g}^{*} \times(k$. $\times \mathfrak{g}^{*}$ by

$$
\begin{aligned}
\operatorname{Coad}^{k}: G \times \mathfrak{g}^{*} \times \stackrel{(k}{.} \times \mathfrak{g}^{*} & \rightarrow \mathfrak{g}^{*} \times \stackrel{(k}{.} \times \mathfrak{g}^{*} \\
\left(g, \mu_{1}, \ldots, \mu_{k}\right) & \mapsto \operatorname{Coad}^{k}\left(g, \mu_{1}, \ldots, \mu_{k}\right)=\left(\operatorname{Coad}\left(g, \mu_{1}\right), \ldots, \operatorname{Coad}\left(g, \mu_{k}\right)\right)
\end{aligned}
$$

which is called the $k$-coadjoint action. 
Let $\mu=\left(\mu_{1}, \ldots, \mu_{k}\right)$ be an element of $\mathfrak{g}^{*} \times \stackrel{(k}{.} \times \mathfrak{g}^{*}$. We will denote by $\mathcal{O}_{\mu}=\mathcal{O}_{\left(\mu_{1}, \ldots, \mu_{k}\right)}$ the $k$-coadjoint orbit at the point $\mu=\left(\mu_{1}, \ldots, \mu_{k}\right)$. If $\xi \in \mathfrak{g}$ then it is easy to prove that the infinitesimal generator of $\mathrm{Coad}^{k}$ associated with $\xi$ is given by

$$
\xi_{\left(\mathfrak{g}^{*} \times\left(k \times \mathfrak{g}^{*}\right)\right.}\left(\nu_{1}, \ldots, \nu_{k}\right)=\left(\xi_{\mathfrak{g}^{*}}\left(\nu_{1}\right), \ldots, \xi_{\mathfrak{g}^{*}}\left(\nu_{k}\right)\right), \quad \text { for }\left(\nu_{1}, \ldots, \nu_{k}\right) \in \mathfrak{g}^{*} \times \stackrel{(k}{.} \times \mathfrak{g}^{*} .
$$

On the other hand, the canonical projection

$$
\operatorname{pr}_{A}: \mathfrak{g}^{*} \times \stackrel{(k}{.} \times \mathfrak{g}^{*} \rightarrow \mathfrak{g}^{*}, \quad\left(\nu_{1}, \ldots, \nu_{k}\right) \mapsto \nu_{A},
$$

induces a smooth map $\pi_{A}: \mathcal{O}_{\mu}=\mathcal{O}_{\left(\mu_{1}, \ldots, \mu_{k}\right)} \rightarrow \mathcal{O}_{\mu_{A}}$ between the $k$-coadjoint orbit $\mathcal{O}_{\mu}$ and the coadjoint orbit $\mathcal{O}_{\mu_{A}}$. Moreover, using (2.6), we deduce that $\pi_{A}$ is a surjective submersion and, in addition,

$$
\cap_{A=1}^{k} \operatorname{Ker}\left(T \pi_{A}\right)=\{0\} .
$$

Now, we consider the family of 2 -forms $\left(\omega_{\mu}^{1}, \ldots, \omega_{\mu}^{k}\right)$ on $\mathcal{O}_{\mu}=\mathcal{O}_{\left(\mu_{1}, \ldots, \mu_{k}\right)}$ given by

$$
\omega_{\mu}^{A}=\pi_{A}^{*}\left(\omega_{\mu_{A}}\right), \quad \text { for every } A \in\{1, \ldots, k\},
$$

where $\omega_{\mu_{A}}$ is the symplectic 2 -form on $\mathcal{O}_{\mu_{A}}$. Then, $\left(\omega_{\mu}^{1}, \ldots, \omega_{\mu}^{k}\right)$ is a $k$-polysymplectic structure on $\mathcal{O}_{\mu}=\mathcal{O}_{\left(\mu_{1}, \ldots, \mu_{k}\right)}($ for more details, see [10, 15]).

\section{K-Poly-Poissson structures}

In this section, we will generalize the notion a k-polysymplectic structure dropping certain non-degeneracy assumption, as Poisson structures generalize symplectic structures. More precisely,

Definition 3.1. A k-poly-Poisson structure on a manifold $M$ is a couple $\left(S, \bar{\Lambda}^{\sharp}\right)$, where $S$ is a vector subbundle of $\left(T_{k}^{1}\right)^{*} M=T^{*} M \oplus . \stackrel{(k}{.} \oplus T^{*} M$ and $\bar{\Lambda}^{\sharp}: S \rightarrow T M$ is a vector bundle morphism which satisfies the following conditions:

i) $\bar{\alpha}\left(\bar{\Lambda}^{\sharp}(\bar{\alpha})\right)=0$ for $\bar{\alpha} \in S$.

ii) If $\bar{\alpha}(\bar{\Lambda} \sharp(\bar{\beta}))=0$ for every $\bar{\beta} \in S$ then $\bar{\Lambda}^{\sharp}(\bar{\alpha})=0$.

iii) If $\bar{\alpha}, \bar{\beta} \in \Gamma(S)$ are sections of $S$ then we have that the following integrability condition holds

$$
\left[\bar{\Lambda}^{\sharp}(\bar{\alpha}), \bar{\Lambda}^{\sharp}(\bar{\beta})\right]=\bar{\Lambda}^{\sharp}\left(\mathcal{L}_{\bar{\Lambda}^{\sharp}(\bar{\alpha})} \bar{\beta}-\mathcal{L}_{\bar{\Lambda}^{\sharp}(\bar{\beta})} \bar{\alpha}-\mathrm{d}\left(\bar{\beta}\left(\bar{\Lambda}^{\sharp}(\bar{\alpha})\right)\right)\right) .
$$

The triple $\left(M, S, \bar{\Lambda}^{\sharp}\right)$ will be called a $k$-poly-Poisson manifold or simply a poly-Poisson manifold.

A $k$-poly-Poisson structure is said to be regular if the vector bundle morphism $\bar{\Lambda}^{\sharp}: S \subseteq$ $\left(T_{k}^{1}\right)^{*} M \rightarrow T M$ has constant rank.

Lemma 3.2. Let $\left(S, \bar{\Lambda}^{\sharp}\right)$ be k-poly-Poisson structure on $M$. Then,

$$
\bar{\alpha}\left(\bar{\Lambda}^{\sharp}(\bar{\beta})\right)=-\bar{\beta}\left(\bar{\Lambda}^{\sharp}(\bar{\alpha})\right), \quad \text { for } \bar{\alpha}, \bar{\beta} \in S .
$$

Proof. By Definition 3.1 i) we have $0=(\bar{\alpha}+\bar{\beta})\left(\bar{\Lambda}^{\sharp}(\bar{\alpha}+\bar{\beta})\right)=\bar{\alpha}\left(\bar{\Lambda}^{\sharp}(\bar{\alpha})\right)+\bar{\alpha}\left(\bar{\Lambda}^{\sharp}(\bar{\beta})\right)+\bar{\beta}\left(\bar{\Lambda}^{\sharp}(\bar{\alpha})\right)+$ $\bar{\beta}\left(\bar{\Lambda}^{\sharp}(\bar{\beta})\right)=\bar{\alpha}\left(\bar{\Lambda}^{\sharp}(\bar{\beta})\right)+\bar{\beta}\left(\bar{\Lambda}^{\sharp}(\bar{\alpha})\right)$ and this implies $\bar{\alpha}\left(\bar{\Lambda}^{\sharp}(\bar{\beta})\right)=-\bar{\beta}\left(\bar{\Lambda}^{\sharp}(\bar{\alpha})\right)$.

As one would expect, polysymplectic manifolds are a particular example of $k$-poly-Poisson manifolds, as it is shown in the following example.

Example 3.3 (Polysymplectic manifolds). Let $(M, \bar{\omega})$ be a polysymplectic manifold. Since $\bar{\omega}^{b}$ is a monomorphism, $S:=\operatorname{Im}\left(\bar{\omega}^{b}\right)$ is a vector subbundle of $\left(T_{k}^{1}\right)^{*} M$. Moreover, we consider

$$
\bar{\Lambda}^{\sharp}:=\left(\bar{\omega}^{b}\right)_{\mid S}^{-1}: S \rightarrow T M .
$$


Clearly, i) in Definition 3.1 is equivalent to i) in Proposition 2.3. On the other hand, if $\bar{\alpha}=$ $\bar{\omega}^{b}(X) \in S$ satisfies

$$
\bar{\alpha}\left(\bar{\Lambda}^{\sharp}(\bar{\beta})\right)=0, \quad \text { for every } \bar{\beta} \in S,
$$

this implies that $\bar{\omega}^{b}(X)=0$. But, using that $\bar{\omega}^{b}$ is a monomorphism, we deduce that $\bar{\Lambda}^{\sharp}(\bar{\alpha})=$ $X=0$. Thus, ii) in Definition 3.1 holds.

Finally, the integrability condition (3.1) is equivalent to iii) in Proposition 2.3. Indeed, if $\bar{\alpha}, \bar{\beta} \in \Gamma(S)$ then, using (2.2) and Lemma 3.2, it follows that

$$
\bar{\omega}^{b}\left[\bar{\Lambda}^{\sharp}(\bar{\alpha}), \bar{\Lambda}^{\sharp}(\bar{\beta})\right]=\mathcal{L}_{\bar{\Lambda} \sharp(\bar{\alpha})} \bar{\beta}-\mathcal{L}_{\bar{\Lambda} \sharp(\bar{\beta})} \bar{\alpha}-\mathrm{d}\left(\bar{\beta}\left(\bar{\Lambda}^{\sharp}(\bar{\alpha})\right)\right) .
$$

which implies that (3.1) holds.

It is not difficult to prove that polysymplectic structures are just poly-Poisson structures satisfying the non-degeneracy condition

$$
\operatorname{Ker}\left(\bar{\Lambda}^{\sharp}\right)=\{0\},
$$

which just says that $\bar{\Lambda}^{\sharp}$ is an isomorphism (note that, using Lemma 3.2, $\operatorname{Ker}\left(\bar{\Lambda}^{\sharp}\right)=\left(\operatorname{Im} \bar{\Lambda}^{\sharp}\right)^{\circ}=$ $\left\{\bar{\alpha} \in\left(T_{k}^{1}\right)^{*} M \mid \bar{\alpha}(X)=0\right.$, for every $\left.\left.X \in \operatorname{Im} \bar{\Lambda}^{\sharp}\right\}\right)$.

It is clear that Poisson manifolds are 1-poly-Poisson manifolds. Indeed, if $\Pi$ is a Poisson structure on $M$ then $\left(S=T^{*} M, \Pi^{\sharp}\right)$ is poly-Poisson, where $\Pi^{\sharp}: T^{*} M \rightarrow T M$ is the vector bundle morphism induced by the Poisson 2-vector $\Pi$. Moreover, it is well known that the generalized distribution

$$
p \in M \rightarrow \Pi^{\sharp}\left(T_{p}^{*} M\right) \subseteq T_{p} M
$$

is a symplectic generalized foliation (see, for instance, [20]). Next, we will prove that a $k$-polyPoisson manifold admits a $k$-polysymplectic generalized foliation.

Theorem 3.4. Let $\left(S, \bar{\Lambda}^{\sharp}\right)$ be a k-poly-Poisson structure on a manifold $M$. Then, $M$ admits a $k$-polysymplectic generalized foliation $\mathcal{F}$ whose characteristic space at the point $p \in M$ is

$$
\mathcal{F}(p)=\bar{\Lambda}^{\sharp}\left(S_{p}\right) \subseteq T_{p} M .
$$

$\mathcal{F}$ is said to be the canonical $k$-polysymplectic foliation of $M$.

Proof. Using that $S$ is a vector subbundle of $\left(T_{k}^{1}\right)^{*} M$, we can choose a local basis $\left\{\bar{\alpha}_{1}, \ldots, \bar{\alpha}_{r}\right\}$ of the space $\Gamma(S)$ of sections of $S$. Thus, it is clear that $\left\{\bar{\Lambda}^{\sharp}\left(\bar{\alpha}_{1}\right), \ldots, \bar{\Lambda}^{\sharp}\left(\bar{\alpha}_{r}\right)\right\}$ is a local generator system of $\mathcal{F}$ which implies that $\mathcal{F}$ is locally finitely generated. Integrability condition of the poly-Poisson structure (Equation (3.1)), makes this distribution involutive. Therefore, $\mathcal{F}$ is a generalized foliation.

Next, let us prove that each leaf $\iota: L \rightarrow M$ of $\mathcal{F}$ is endowed with a $k$-polysymplectic structure $\bar{\omega}^{b}: T L \rightarrow\left(T_{k}^{1}\right)^{*} L$. Given $X \in T_{p} L=\mathcal{F}(p)$ there exists $\bar{\alpha} \in S_{p}$ such that $\bar{\Lambda}^{\sharp}(\bar{\alpha})=X$. Then,

$$
\bar{\omega}^{b}(X)=\iota^{*} \bar{\alpha} .
$$

First, we will show that $\bar{\omega}^{b}$ is well defined. Assume that $X=\bar{\Lambda}^{\sharp}(\bar{\alpha})=\bar{\Lambda}^{\sharp}(\bar{\beta})$. Then $\bar{\Lambda}^{\sharp}(\bar{\alpha}-\bar{\beta})=0$ and, using Lemma 3.2,

$$
\begin{aligned}
0 & =\bar{\gamma}\left(\overline{\Lambda^{\sharp}}(\bar{\alpha}-\bar{\beta})\right) \\
& =-(\bar{\alpha}-\bar{\beta})\left(\overline{\Lambda^{\sharp}}(\bar{\gamma})\right)
\end{aligned}
$$

for any $\bar{\gamma} \in S$. As a consequence, $\iota^{*}(\bar{\alpha}-\bar{\beta})=0$.

It is easy to see that $\bar{\omega}^{b}$ is injective. Indeed, if $X=\bar{\Lambda}^{\sharp}(\bar{\alpha})$ then $\bar{\omega}^{b}(X)=0$ is equivalent to $\bar{\alpha}\left(\bar{\Lambda}^{\sharp}(\bar{\gamma})\right)=0$ for all $\bar{\gamma} \in S$. But condition ii) of Definition 3.1 implies that $X=\bar{\Lambda}^{\sharp}(\bar{\alpha})=0$. 
To prove the integrability condition (2.2), it is enough to prove the relation for a generating subset. Choose $\bar{\alpha}_{1}, \ldots, \bar{\alpha}_{r}$ a local basis of $\Gamma(S)$ and denote $X_{i}=\bar{\Lambda}^{\sharp}\left(\bar{\alpha}_{i}\right), i=1, \ldots, r$, which generates the foliation $\mathcal{F}$. Taking two elements $X_{i}=\bar{\Lambda}^{\sharp}\left(\bar{\alpha}_{i}\right), X_{j}=\bar{\Lambda}^{\sharp}\left(\bar{\alpha}_{j}\right)$, using (3.1), we have

$$
\left[X_{i}, X_{j}\right]=\bar{\Lambda}^{\sharp}\left(\mathcal{L}_{\bar{\Lambda}^{\sharp}\left(\bar{\alpha}_{i}\right)} \bar{\alpha}_{j}-\mathcal{L}_{\bar{\Lambda}^{\sharp}\left(\bar{\alpha}_{j}\right)} \bar{\alpha}_{i}-\mathrm{d}\left(\bar{\alpha}_{j}\left(\bar{\Lambda}^{\sharp}\left(\bar{\alpha}_{i}\right)\right)\right)\right) .
$$

Applying $\bar{\omega}^{b}$ to both sides of the equation, from the fact that $\bar{\omega} \circ \bar{\Lambda}^{\sharp}(\bar{\alpha})=\iota^{*} \bar{\alpha}, \iota$ being the inclusion from the leaf into the manifold $M$, this is just

$$
\bar{\omega}^{b}\left(\left[X_{i}, X_{j}\right]\right)=\iota^{*}\left(\mathcal{L}_{\bar{\Lambda}^{\sharp}\left(\bar{\alpha}_{i}\right)} \bar{\alpha}_{j}-\mathcal{L}_{\bar{\Lambda}^{\sharp}\left(\bar{\alpha}_{j}\right)} \bar{\alpha}_{i}-\mathrm{d}\left(\bar{\alpha}_{j}\left(\bar{\Lambda}^{\sharp}\left(\bar{\alpha}_{i}\right)\right)\right)\right),
$$

and, from Equation (3.2) and the properties of Lie derivative and pull-back, we have

$$
\iota^{*}\left(\mathcal{L}_{\bar{\Lambda}^{\sharp}\left(\bar{\alpha}_{i}\right)} \bar{\alpha}_{j}-\mathcal{L}_{\bar{\Lambda}^{\sharp}\left(\bar{\alpha}_{j}\right)} \bar{\alpha}_{i}-\mathrm{d}\left(\bar{\alpha}_{j}\left(\bar{\Lambda}^{\sharp}\left(\bar{\alpha}_{i}\right)\right)\right)\right)=\mathcal{L}_{X_{i}} \bar{\omega}^{b}\left(X_{j}\right)-\mathcal{L}_{X_{j}} \bar{\omega}^{b}\left(X_{i}\right)-\mathrm{d}\left(\bar{\omega}^{b}\left(X_{j}\right)\left(X_{i}\right)\right),
$$

so the integrability condition for $\bar{\omega}$ holds.

Example 3.5. Let $(M, \bar{\omega})$ be a connected polysymplectic manifold and consider the corresponding poly-Poisson manifold $\left(M, S, \bar{\Lambda}^{\sharp}\right)$. Then, from Example 3.3. the distribution $\bar{\Lambda}^{\sharp}(S)$ is just $T M$. Thus, there is only one leaf, the manifold $M$, and the poly-symplectic structure on it is just $\bar{\omega}$.

Next, we will prove a converse of Theorem 3.4.

Let $M$ be a smooth manifold, $S$ a vector subbundle of $\left(T_{k}^{1}\right)^{*} M$ and $\bar{\Lambda}^{\sharp}: S \rightarrow T M$ a vector bundle morphism such that conditions i) and ii) in Definition 3.1 hold. If $p$ is a point of $M$, we can define the nondegenerate $\mathbb{R}^{k}$-valued 2-form $\bar{\omega}(p): \bar{\Lambda}^{\sharp}\left(S_{p}\right) \times \bar{\Lambda} \sharp\left(S_{p}\right) \rightarrow \mathbb{R}^{k}$ on the vector space $\bar{\Lambda} \sharp\left(S_{p}\right)$ given by

$$
\bar{\omega}(p)\left(\bar{\Lambda}^{\sharp}\left(\bar{\alpha}_{p}\right), \bar{\Lambda}^{\sharp}\left(\bar{\beta}_{p}\right)\right)=\bar{\alpha}_{p}\left(\bar{\Lambda}^{\sharp}\left(\bar{\beta}_{p}\right)\right), \text { for } \bar{\alpha}_{p}, \bar{\beta}_{p} \in S_{p} .
$$

In fact, since $\bar{\alpha}\left(\bar{\Lambda}^{\sharp}(\bar{\beta})\right)=-\bar{\beta}\left(\bar{\Lambda}^{\sharp}(\bar{\alpha})\right)$ for $\bar{\alpha}, \bar{\beta} \in S$, we deduce that $\bar{\omega}(p)$ is well-defined.

Now, assume that the generalized distribution $\mathcal{F}$ on $M$ defined by

$$
p \in M \rightarrow \mathcal{F}(p)=\bar{\Lambda}^{\sharp}\left(S_{p}\right) \subseteq T_{p} M
$$

is involutive. Then, since $\mathcal{F}$ is locally finitely generated, we have that $\mathcal{F}$ is a generalized foliation.

In addition, if $L$ is a leaf of $\mathcal{F}$ then the restriction of $\bar{\omega}$ to $L$ induces a nondegenerate $\mathbb{R}^{k}$ valued 2 -form $\bar{\omega}_{L}$ on $L$. Therefore, if $\bar{\omega}_{L}$ is closed we deduce that $\bar{\omega}_{L}$ defines a $k$-polysymplectic structure on $L$.

We will see that if this condition holds for every leaf $L$ then the couple $\left(S, \bar{\Lambda}^{\sharp}\right)$ is a $k$-polyPoisson structure on $M$. Indeed, the result follows using Proposition 2.3 (more precisely Eq. (2.2)) and the fact that

$$
\bar{\omega}_{L}\left(\bar{\Lambda}^{\sharp}(\bar{\alpha})_{\mid L}\right)=\iota^{*} \bar{\alpha}, \quad \text { for } \bar{\alpha} \in \Gamma(S),
$$

where $\iota: L \rightarrow M$ is the canonical inclusion and we also denote by $\bar{\omega}_{L}$ the vector bundle morphism between $T L$ and $\left(T_{k}^{1}\right)^{*} L$ induced by the $\mathbb{R}^{k}$-valued 2 -form $\bar{\omega}_{L}$.

In conclusion, we have proved the following result.

Theorem 3.6. Let $S$ be a vector subbundle of $\left(T_{k}^{1}\right)^{*} M$ and $\bar{\Lambda}^{\sharp}: S \subseteq\left(T_{k}^{1}\right)^{*} M \rightarrow T M$ a vector bundle morphism such that:

i) $\bar{\alpha}\left(\bar{\Lambda}^{\sharp}(\bar{\alpha})\right)=0$, for $\bar{\alpha} \in S$, and

ii) if $\bar{\alpha}\left(\bar{\Lambda}^{\sharp}(\bar{\beta})\right)=0$ for every $\bar{\beta} \in S$ then $\bar{\Lambda} \sharp(\bar{\alpha})=0$.

Assume also that the generalized distribution

$$
p \in M \rightarrow \mathcal{F}(p)=\bar{\Lambda}^{\sharp}\left(S_{p}\right) \subseteq T_{p} M
$$


is involutive and for every leaf $L$ the corresponding $\mathbb{R}^{k}$-valued 2 -form $\bar{\omega}_{L}$ on $L$ (induced by the morphism $\left.\bar{\Lambda}^{\sharp}\right)$ is closed. Then, the couple $\left(S, \bar{\Lambda}^{\sharp}\right)$ is a k-poly-Poisson structure on $M$ and $\mathcal{F}$ is the canonical $k$-polysymplectic foliation of $M$.

Next, we will discuss a construction which allows to obtain some examples of $k$-poly-Poisson manifolds. This can be seen as a foliated version of the one presented in Example 2.5. For this purpose, we will use Theorem 3.6.

Let $M_{A}$ be a Poisson manifold, with $A \in\{1, \ldots, k\}$, and $\pi_{A}: M \rightarrow M_{A}$ a fibration. Denote by $\Lambda^{A}$ the Poisson 2-vector of $M_{A}$ and by $\mathcal{F}_{A}$ the symplectic foliation in $M_{A}$. Assume that $\mathcal{F}$ is a generalized foliation on $M$ such that

i) For every $p \in M$

$$
\left(T_{p} \pi_{A}\right)(\mathcal{F}(p))=\mathcal{F}_{A}\left(\pi_{A}(p)\right) .
$$

ii) For every $p \in M$

$$
\left(\cap_{A=1}^{k} \operatorname{Ker}\left(T_{p} \pi_{A}\right)\right) \cap \mathcal{F}(p)=\{0\} .
$$

We will show that every leaf $L$ of $\mathcal{F}$ admits a $k$-polysymplectic structure. We will proceed as in Section 2 .

Let $p$ be a point of $M, L$ the leaf of $\mathcal{F}$ over the point $p$ and $L_{A}$ the symplectic leaf of $M_{A}$ over the point $\pi_{A}(p)$, for every $A \in\{1, \ldots, k\}$. First of all, we will define a $k$-polysymplectic structure $\left(\omega_{L}^{1}(p), \ldots, \omega_{L}^{k}(p)\right)$ on the vector space $\mathcal{F}(p)=T_{p} L$. In fact, if $\Omega_{L_{A}}\left(\pi_{A}(p)\right)$ is the symplectic 2 -form on the vector space $\mathcal{F}_{A}\left(\pi_{A}(p)\right)=T_{\pi_{A}(p)} L_{A}$ then

$$
\omega_{L}^{A}(p)=\left(T_{p}^{*} \pi_{A}\right)\left(\Omega_{L_{A}}\left(\pi_{A}(p)\right)\right) .
$$

Remark 3.7. If $u, v \in \mathcal{F}(p)=T_{p} L$, we have that

$$
\left(T_{p} \pi_{A}\right)(u)=\Lambda_{A}^{\sharp}\left(\widetilde{\alpha}_{A}\right),\left(T_{p} \pi_{A}\right)(v)=\Lambda_{A}^{\sharp}\left(\widetilde{\beta}_{A}\right), \quad \text { with } \widetilde{\alpha}_{A}, \widetilde{\beta}_{A} \in T_{\pi_{A}(p)}^{*} M_{A} .
$$

Thus, since

we obtain that

$$
\Omega_{L_{A}}\left(\pi_{A}(p)\right)\left(\Lambda_{A}^{\sharp}\left(\widetilde{\alpha}_{A}\right), \Lambda_{A}^{\sharp}\left(\widetilde{\beta}_{A}\right)\right)=\widetilde{\alpha}_{A}\left(\Lambda_{A}^{\sharp}\left(\widetilde{\beta}_{A}\right)\right),
$$

$$
\omega_{L}^{A}(u, v)=\widetilde{\alpha}_{A}\left(\Lambda_{A}^{\sharp}\left(\widetilde{\beta}_{A}\right)\right) .
$$

From (3.3), (3.4) and (3.5), it follows that $\left(\omega_{L}^{1}(p), \ldots, \omega_{L}^{k}(p)\right)$ is a $k$-polysymplectic structure on the vector space $\mathcal{F}(p)=T_{p} L$, that is,

$$
\cap_{A=1}^{k} \operatorname{Ker}\left(\omega_{L}^{A}(p)\right)=\{0\} .
$$

Therefore, $L$ admits an almost $k$-polysymplectic structure $\left(\omega_{L}^{1}, \ldots, \omega_{L}^{k}\right)$, i.e., $\omega_{L}^{A}$ is a 2 -form on $L$, for every $A \in\{1, \ldots, k\}$, and

$$
\cap_{A=1}^{k} \operatorname{Ker} \omega_{L}^{A}=\{0\} .
$$

In addition, using (3.3), (3.4) and the fact that $\Omega_{L_{A}}$ is a closed 2-form on $L_{A}$, we deduce that the 2 -forms $\omega_{L}^{A}$ are also closed and $\left(\omega_{L}^{1}, \ldots, \omega_{L}^{k}\right)$ is a $k$-polysymplectic structure on $L$. Consequently, $\mathcal{F}$ is a $k$-polysymplectic foliation.

Now, we will introduce a vector subspace $S(p)$ of $\left(T_{k}^{1}\right)_{p}^{*} M$ and a linear epimorphism $\bar{\Lambda}^{\sharp}: S(p) \subseteq$ $\left(T_{k}^{1}\right)^{*} M \rightarrow \mathcal{F}(p) \subseteq T_{p} M$.

From (3.3) and (3.4), we have that the linear map $T_{p} \pi: \mathcal{F}(p) \rightarrow \mathcal{F}_{1}\left(\pi_{1}(p)\right) \times \ldots \times \mathcal{F}_{k}\left(\pi_{k}(p)\right)$ given by

$$
\left(T_{p} \pi\right)(u)=\left(\left(T_{p} \pi_{1}\right)(u), \ldots,\left(T_{p} \pi_{k}\right)(u)\right), \quad \text { for } u \in \mathcal{F}(p),
$$

is a linear monomorphism. 
Next, we define

$$
\begin{array}{r}
S(p)=\left\{\left(\left(T_{p}^{*} \pi_{1}\right)\left(\widetilde{\alpha}_{1}\right), \ldots,\left(T_{p}^{*} \pi_{k}\right)\left(\widetilde{\alpha}_{k}\right)\right) \in\left(T_{k}^{1}\right)_{p}^{*} M \mid \exists u \in \mathcal{F}(p)\right. \text { and } \\
\left.\left(T_{p} \pi\right)(u)=\left(\Lambda_{1}^{\sharp}\left(\widetilde{\alpha}_{1}\right), \ldots, \Lambda_{k}^{\sharp}\left(\widetilde{\alpha}_{k}\right)\right)\right\}
\end{array}
$$

and the linear epimorphism $\bar{\Lambda}^{\sharp}: S(p) \subseteq\left(T_{k}^{1}\right)_{p}^{*} M \rightarrow \mathcal{F}(p)$ by

$$
\bar{\Lambda}^{\sharp}\left(\left(T_{p}^{*} \pi_{1}\right)\left(\widetilde{\alpha}_{1}\right), \ldots,\left(T_{p}^{*} \pi_{k}\right)\left(\widetilde{\alpha}_{k}\right)\right)=u \text {, if }\left(T_{p} \pi\right)(u)=\left(\Lambda_{1}^{\sharp}\left(\widetilde{\alpha}_{1}\right), \ldots, \Lambda_{k}^{\sharp}\left(\widetilde{\alpha}_{k}\right)\right) .
$$

Note that $\bar{\Lambda}^{\sharp}$ is well-defined because $T_{p} \pi$ is injective. Moreover, we will see that the couple $\left(S(p), \bar{\Lambda}^{\sharp}\right)$ satisfies conditions i) and ii) in Theorem 3.6 .

First, if

it follows that

$$
\bar{\alpha}=\left(\alpha_{1}, \ldots, \alpha_{k}\right)=\left(\left(T_{p}^{*} \pi_{1}\right)\left(\widetilde{\alpha}_{1}\right), \ldots,\left(T_{p}^{*} \pi_{k}\right)\left(\widetilde{\alpha}_{k}\right)\right) \in S(p)
$$

$$
\alpha_{A}\left(\bar{\Lambda}^{\sharp}(\bar{\alpha})\right)=\widetilde{\alpha}_{A}\left(\left(T_{p} \pi_{A}\right)(u)\right)=\widetilde{\alpha}_{A}\left(\Lambda_{A}^{\sharp}\left(\widetilde{\alpha}_{A}\right)\right)=0 \text {, for all } A .
$$

Furthermore, if for every $\bar{\beta} \in S(p)$

$$
\bar{\alpha}\left(\bar{\Lambda}^{\sharp}(\bar{\beta})\right)=0
$$

then we will prove that $\bar{\Lambda}^{\sharp}(\bar{\alpha})=0$. Indeed, suppose that $u=\bar{\Lambda}^{\sharp}(\bar{\alpha}) \in \mathcal{F}(p)$. This implies that $\left(T_{p} \pi_{A}\right)(u)=\Lambda_{A}^{\sharp}\left(\widetilde{\alpha}_{A}\right)$. Now, let $v$ be an arbitrary element of $\mathcal{F}(p)$. We have that

$$
\left(T_{p} \pi_{A}\right)(v)=\Lambda_{A}^{\sharp}\left(\widetilde{\beta}_{A}\right), \quad \text { with } \widetilde{\beta}_{A} \in T_{\pi_{A}(p)}^{*} M_{A},
$$

for every $A \in\{1, \ldots, k\}$. Thus,

$$
\bar{\beta}=\left(\left(T_{p}^{*} \pi_{1}\right)\left(\widetilde{\beta}_{1}\right), \ldots,\left(T_{p}^{*} \pi_{k}\right)\left(\widetilde{\beta}_{k}\right)\right) \in S(p)
$$

and

$$
0=\alpha_{A}\left(\bar{\Lambda}^{\sharp}(\bar{\beta})\right)=\widetilde{\alpha}_{A}\left(\Lambda_{A}^{\sharp}\left(\widetilde{\beta}_{A}\right)\right), \quad \text { for every } A \in\{1, \ldots, k\} .
$$

Therefore, using (3.6), it is deduced that

$$
0=\omega_{L}^{A}(p)(u, v), \quad \text { for every } A \in\{1, \ldots, k\} .
$$

Consequently, from (3.7), we obtain that

$$
0=u=\bar{\Lambda}^{\sharp}(\bar{\alpha}) .
$$

So, if the assignment

$$
p \in M \mapsto S(p) \subseteq\left(T_{k}^{1}\right)_{p}^{*} M
$$

defines a vector subbundle of $\left(T_{k}^{1}\right)^{*} M$ then, using Theorem 3.6, it follows that the couple $\left(S, \bar{\Lambda}^{\sharp}\right)$ is a $k$-poly-Poisson structure on $M$ with canonical $k$-poly-symplectic foliation $\mathcal{F}$.

We will see that this construction works in some interesting examples.

Example 3.8 (The product of Poisson manifolds). Let $\left(M_{A}, \Lambda_{A}\right)$ be a Poisson manifold, with $A \in\{1, \ldots, k\}$, and $M$ the product manifold $M=M_{1} \times \ldots \times M_{k}$. Denote by $\pi_{A}: M \rightarrow M_{A}$ the canonical projection and by $\mathcal{F}_{A}$ the symplectic foliation in $M_{A}$.

We consider the foliation $\mathcal{F}$ in $M$ whose characteristic space at the point $p=\left(p_{1}, \ldots, p_{k}\right) \in$ $M_{1} \times \ldots \times M_{k}=M$ is

$$
\mathcal{F}(p)=\mathcal{F}\left(p_{1}, \ldots, p_{k}\right)=\mathcal{F}_{1}\left(p_{1}\right) \times \ldots \times \mathcal{F}_{k}\left(p_{k}\right) \subseteq T_{p_{1}} M_{1} \times \ldots \times T_{p_{k}} M_{k} \cong T_{p} M .
$$

It is clear that (3.3) and (3.4) hold. Thus, the leaves of $\mathcal{F}$ admit a $k$-polysymplectic structure. If $p=\left(p_{1}, \ldots, p_{k}\right) \in M_{1} \times \ldots \times M_{k}=M$ then, in this case, the map

$$
T_{p} \pi: \mathcal{F}(p) \rightarrow \mathcal{F}_{1}\left(p_{1}\right) \times \ldots \times \mathcal{F}_{k}\left(p_{k}\right)
$$

is a linear isomorphism. This implies that

$$
S(p)=\left\{\left(\left(T_{p}^{*} \pi_{1}\right)\left(\widetilde{\alpha}_{1}\right), \ldots,\left(T_{p}^{*} \pi_{k}\right)\left(\widetilde{\alpha}_{k}\right)\right) \in\left(T_{k}^{1}\right)_{p}^{*} M \mid \widetilde{\alpha}_{A} \in T_{\pi_{A}(p)}^{*} M_{A} \text {, for all } A\right\} .
$$

In other words,

$$
S(p) \cong T_{\pi_{1}(p)}^{*} M_{1} \times \ldots \times T_{\pi_{k}(p)}^{*} M_{k} \cong T_{p}^{*} M .
$$


Therefore, the assignment

$$
p \in M \mapsto S(p) \subseteq\left(T_{k}^{1}\right)_{p}^{*} M
$$

is a vector subbundle of $\left(T_{k}^{1}\right)_{p}^{*} M$ of rank $m$, with $m=\sum_{A=1}^{k} m_{A}$ and $m_{A}=\operatorname{dim} M_{A}$, which may be identified with the cotangent bundle $T^{*} M \rightarrow M$ to $M$.

Under this identification, the vector bundle morphism

$$
\bar{\Lambda}^{\sharp}: S \cong T^{*} M=T^{*} M_{1} \times \ldots \times T^{*} M_{k} \rightarrow \mathcal{F} \subseteq T M=T M_{1} \times \ldots T M_{k}
$$

is given by

for $\left(\widetilde{\alpha}_{1}, \ldots, \widetilde{\alpha}_{k}\right) \in T^{*} M_{1} \times \ldots \times T^{*} M_{k}$.

$$
\bar{\Lambda}^{\sharp}\left(\widetilde{\alpha}_{1}, \ldots, \widetilde{\alpha}_{k}\right)=\left(\Lambda_{1}^{\sharp}\left(\widetilde{\alpha}_{1}\right), \ldots, \Lambda_{k}^{\sharp}\left(\widetilde{\alpha}_{k}\right)\right),
$$

Example 3.9 (The Whitney sum $E_{k}^{*}=E^{*} \oplus . \stackrel{(k}{*} \oplus E^{*}$, with $E$ a Lie algebroid). Let $\tau_{E}: E \rightarrow Q$ be a Lie algebroid of rank $n$ over a manifold $Q$ of dimension $m, \Lambda_{E^{*}}$ the fiberwise linear Poisson structure on the dual bundle $E^{*}$ to $E$ and $\mathcal{F}_{E^{*}}$ the symplectic foliation associated with $\Lambda_{E^{*}}$ (see Appendix).

We consider the Whitney sum $E_{k}^{*}=E^{*} \oplus \ldots \oplus E^{*}$. It is a vector bundle over $Q$ whose fiber at the point $q \in Q$ is $\left(E_{k}^{*}\right)_{q}=E_{q}^{*} \times \ldots \times E_{q}^{*}$. We will denote by $\tau_{E_{k}^{*}}: E_{k}^{*} \rightarrow Q$ the vector bundle projection.

If $\bar{\alpha}=\left(\alpha_{1}, \ldots, \alpha_{k}\right) \in\left(E_{k}^{*}\right)_{q}$ we have that $T_{\bar{\alpha}} E_{k}^{*}=\left\{\left(\widetilde{v}_{1}, \ldots, \widetilde{v}_{k}\right) \in T_{\alpha_{1}} E^{*} \times \ldots \times T_{\alpha_{k}} E^{*} \mid\left(T_{\alpha_{A}} \tau_{E^{*}}\right)\left(\widetilde{v}_{A}\right)=\left(T_{\alpha_{B}} \tau_{E^{*}}\right)\left(\widetilde{v}_{B}\right)\right.$, for all $A$ and $\left.B\right\}$, where $\tau_{E^{*}}: E^{*} \rightarrow Q$ is the vector bundle projection. Thus, if $\widetilde{\alpha}_{A} \in T_{\alpha_{A}}^{*} E^{*}$ for every $A$ then, using (A.3) (see Appendix), it follows that

$$
\left(\Lambda_{E^{*}}^{\sharp}\left(\widetilde{\alpha}_{1}\right), \ldots, \Lambda_{E^{*}}^{\sharp}\left(\widetilde{\alpha}_{k}\right)\right) \in T_{\bar{\alpha}} E_{k}^{*}
$$

if and only if

$$
\widetilde{\alpha}_{A} \circ{ }_{\alpha_{A}}^{\mathrm{v}} \circ \rho_{E \mid T_{q}^{*} Q}^{*}=\widetilde{\alpha}_{B} \circ{ }_{\alpha_{B}}^{\mathrm{v}} \circ \rho_{E \mid T_{q}^{*} Q}^{*}, \quad \text { for all } A \text { and } B,
$$

$\rho_{E}: E \rightarrow T Q$ being the anchor map of $E, \rho_{E}^{*}: T^{*} Q \rightarrow E^{*}$ the dual morphism of $\rho_{E}$ and ${ }_{\alpha_{C}}^{\mathrm{v}}: E_{q}^{*} \rightarrow T_{\alpha_{C}} E_{q}^{*}$ the canonical isomorphism between $E_{q}^{*}$ and $T_{\alpha_{C}} E_{q}^{*}$, for every $C \in\{1, \ldots, k\}$.

Denote by $\pi_{A}: E_{k}^{*} \rightarrow E^{*}$ the canonical projection given by

$$
\pi_{A}\left(\alpha_{1}, \ldots, \alpha_{k}\right)=\alpha_{A}, \quad \text { for }\left(\alpha_{1}, \ldots, \alpha_{k}\right) \in E_{k}^{*} .
$$

Then, it is clear that $\pi_{A}: E_{k}^{*} \rightarrow E^{*}$ is a surjective submersion. In fact,

$$
\left(T_{\bar{\alpha}} \pi_{A}\right)\left(\widetilde{v}_{1}, \ldots, \widetilde{v}_{k}\right)=\widetilde{v}_{A}, \quad \text { for }\left(\widetilde{v}_{1}, \ldots, \widetilde{v}_{k}\right) \in T_{\bar{\alpha}} E_{k}^{*} .
$$

Now, let $\mathcal{F}$ be the generalized distribution on $E_{k}^{*}$ whose characteristic space at the point $\bar{\alpha}=$ $\left(\alpha_{1}, \ldots, \alpha_{k}\right) \in\left(E_{k}^{*}\right)_{q}$ is

$$
\begin{array}{r}
\mathcal{F}(\bar{\alpha})=\left\{\left(\Lambda_{E^{*}}^{\sharp}\left(\widetilde{\alpha}_{1}\right), \ldots, \Lambda_{E^{*}}^{\sharp}\left(\widetilde{\alpha}_{k}\right)\right) \in T_{\alpha_{1}} E^{*} \times \ldots \times T_{\alpha_{k}} E^{*} \mid \widetilde{\alpha}_{A} \in T_{\alpha_{A}}^{*} E^{*}\right. \text { and } \\
\left.\widetilde{\alpha}_{A} \circ{ }_{\pi_{A}(\bar{\alpha})}^{\mathrm{v}}=\widetilde{\alpha}_{B} \circ{ }_{\pi_{B}(\bar{\alpha})}^{\mathrm{v}}\right\}
\end{array}
$$

Note that the condition $\widetilde{\alpha}_{A} \circ{ }_{\pi_{A}(\bar{\alpha})}^{\mathrm{v}}=\widetilde{\alpha}_{B} \circ{ }_{\pi_{B}(\bar{\alpha})}^{\mathrm{v}}$, for all $A$ and $B$, implies that

$$
\left(\Lambda_{E^{*}}^{\sharp}\left(\widetilde{\alpha}_{1}\right), \ldots, \Lambda_{E^{*}}^{\sharp}\left(\widetilde{\alpha}_{k}\right)\right) \in T_{\bar{\alpha}} E_{k}^{*} .
$$

Next, we will prove that $\mathcal{F}$ is a generalized foliation on $E_{k}^{*}$. First of all, we will see that $\mathcal{F}$ is locally finitely generated.

Let $\left(q^{i}\right)$ be local coordinates on an open subset $U \subseteq Q$ and $\left\{e_{\alpha}\right\}$ be a local basis of $\Gamma\left(\tau_{E}^{-1}(U)\right)$. Denote by $\left(q^{i}, p_{\alpha}\right)$ the corresponding local coordinates on $E^{*}$. Then, we have local coordinates $\left(q^{i}, p_{\alpha}^{A}\right)$ on $E_{k}^{*}=E^{*} \oplus \ldots \oplus E^{*}$. In fact, if $\bar{\alpha} \in \tau_{E_{k}^{*}}^{-1}(U)$

$$
q^{i}(\bar{\alpha})=q^{i}\left(\tau_{E_{k}^{*}}(\bar{\alpha})\right), \quad p_{\alpha}^{A}(\bar{\alpha})=p_{\alpha}\left(\pi_{A}(\bar{\alpha})\right),
$$

for $i \in\{1, \ldots, m\}, \alpha \in\{1, \ldots, n\}$ and $A \in\{1, \ldots, k\}$. 
Moreover,

$$
\begin{aligned}
& \left\{\left(0, \ldots, \Lambda_{E^{*}}^{\sharp A)}\left(\mathrm{d} q^{i}\right), \ldots, 0\right),\left(\Lambda_{E^{*}}^{\sharp}\left(\mathrm{d} p_{\alpha}\right), \ldots, \Lambda_{E^{*}}^{\sharp}\left(\mathrm{d} p_{\alpha}\right)\right) \mid i \in\{1, \ldots, m\}, \alpha \in\{1, \ldots, n\}\right. \\
& \text { and } A \in\{1, \ldots, k\}\}
\end{aligned}
$$

is a local generator system of $\mathcal{F}$. Thus, using (A.2), it follows that

$$
\left\{\rho_{\beta}^{i} \frac{\partial}{\partial p_{\beta}^{A}}, \rho_{\alpha}^{i} \frac{\partial}{\partial q^{i}}+\mathcal{C}_{\alpha \beta}^{\gamma} p_{\gamma}^{B} \frac{\partial}{\partial p_{\beta}^{B}} \mid i \in\{1, \ldots, m\}, \alpha \in\{1, \ldots, n\} \text { and } A \in\{1, \ldots, k\}\right\}
$$

is a local generator system of $\mathcal{F}$, where $\left(\rho_{\beta}^{i}, \mathrm{C}_{\alpha \beta}^{\gamma}\right)$ are the local structure functions of $E$. Therefore, $\mathcal{F}$ is locally finitely generated.

On the other hand, from (A.1), we deduce that

$$
\begin{aligned}
{\left[\rho_{\alpha}^{i} \frac{\partial}{\partial p_{\alpha}^{A}}, \rho_{\beta}^{j} \frac{\partial}{\partial p_{\beta}^{B}}\right] } & =0 \\
{\left[\rho_{\alpha}^{i} \frac{\partial}{\partial p_{\alpha}^{A}}, \rho_{\beta}^{j} \frac{\partial}{\partial q^{j}}+\mathcal{C}_{\beta \gamma}^{\mu} p_{\mu}^{B} \frac{\partial}{\partial p_{\gamma}^{B}}\right] } & =-\frac{\partial \rho_{\beta}^{i}}{\partial q^{j}}\left(\rho_{\gamma}^{j} \frac{\partial}{\partial p_{\gamma}^{A}}\right) \\
{\left[\rho_{\alpha}^{i} \frac{\partial}{\partial q^{i}}+\mathcal{C}_{\alpha \beta}^{\gamma} p_{\gamma}^{A} \frac{\partial}{\partial p_{\beta}^{A}}, \rho_{\mu}^{j} \frac{\partial}{\partial q^{j}}+\mathcal{C}_{\mu \nu}^{\theta} p_{\theta}^{B} \frac{\partial}{\partial p_{\nu}^{B}}\right] } & =\mathcal{C}_{\alpha \mu}^{\nu}\left(\rho_{\nu}^{i} \frac{\partial}{\partial q^{i}}-\mathcal{C}_{\beta \nu}^{\theta} p_{\theta}^{A} \frac{\partial}{\partial p_{\beta}^{A}}\right)-p_{\theta}^{A} \frac{\partial \mathcal{C}_{\alpha \mu}^{\theta}}{\partial q^{i}}\left(\rho_{\beta}^{i} \frac{\partial}{\partial p_{\beta}^{A}}\right) .
\end{aligned}
$$

This implies that $\mathcal{F}$ is involutive. Consequently, $\mathcal{F}$ is a generalized foliation on $E_{k}^{*}$.

Now, we will prove that conditions (3.3) and (3.4) hold for the foliation $\mathcal{F}$. We have that

$$
\mathcal{F}_{E^{*}}(\alpha)=\Lambda_{E^{*}}^{\sharp}\left(T_{\alpha}^{*} E^{*}\right), \quad \text { for } \alpha \in E^{*} .
$$

Thus, from (3.9) and (3.10), it follows that

$$
\left(T_{\bar{\alpha}} \pi_{A}\right)(\mathcal{F}(\bar{\alpha})) \subseteq \mathcal{F}_{E^{*}}\left(\pi_{A}(\bar{\alpha})\right), \quad \text { for } \bar{\alpha} \in E_{k}^{*} \text { and } A \in\{1, \ldots, k\} .
$$

Moreover, if $A \in\{1, \ldots, k\}$ and $\widetilde{v}_{A} \in \mathcal{F}_{E^{*}}\left(\pi_{A}(\bar{\alpha})\right)$ then

$$
\widetilde{v}_{A}=\Lambda_{E^{*}}^{\sharp}\left(\widetilde{\alpha}_{A}\right), \quad \text { with } \widetilde{\alpha}_{A} \in T_{\pi_{A}(\bar{\alpha})}^{*} E^{*} .
$$

In addition, it is easy to prove that we can choose $\widetilde{\alpha}_{B} \in T_{\pi_{B}(\bar{\alpha})}^{*} E^{*}$, with $B \in\{1, \ldots, k\}$, satisfying

$$
\widetilde{\alpha}_{B} \circ \stackrel{\mathrm{v}}{\pi_{B}(\bar{\alpha})}=\widetilde{\alpha}_{A} \circ{\stackrel{\mathrm{v}}{\pi_{A}(\bar{\alpha})}}^{*}
$$

Therefore, $\left(\Lambda_{E^{*}}^{\sharp}\left(\widetilde{\alpha}_{1}\right), \ldots, \Lambda_{E^{*}}^{\sharp}\left(\widetilde{\alpha}_{k}\right)\right) \in \mathcal{F}(\bar{\alpha})$ and, using (3.9)), we deduce that

$$
\left(T_{\bar{\alpha}} \pi_{A}\right)\left(\Lambda_{E^{*}}^{\sharp}\left(\widetilde{\alpha}_{1}\right), \ldots, \Lambda_{E^{*}}^{\sharp}\left(\widetilde{\alpha}_{k}\right)\right)=\Lambda_{E^{*}}^{\sharp}\left(\widetilde{\alpha}_{A}\right)=\widetilde{v}_{A} .
$$

This proves (3.3) for the foliation $\mathcal{F}$.

On the other hand, using again (3.9), we obtain that

$$
\cap_{A=1}^{k} \operatorname{Ker}\left(T_{\bar{\alpha}} \pi_{A}\right)=\{0\}
$$

and, as a consequence, (3.4) also holds for the foliation $\mathcal{F}$.

Next, we will see that the assignment

$$
\bar{\alpha} \in E_{k}^{*} \rightarrow S(\bar{\alpha}) \subseteq\left(T_{k}^{1}\right)_{\bar{\alpha}}^{*} E_{k}^{*}
$$

defines a vector subbundle of $\left(T_{k}^{1}\right)^{*} E_{k}^{*}$.

In fact, if $\bar{\alpha} \in E_{k}^{*}$ then, from (3.8), we have that

$$
\begin{array}{r}
S(\bar{\alpha})=\left\{\left(\left(T_{\bar{\alpha}}^{*} \pi_{1}\right)\left(\widetilde{\alpha}_{1}\right), \ldots,\left(T_{\bar{\alpha}}^{*} \pi_{k}\right)\left(\widetilde{\alpha}_{k}\right)\right) \in\left(T_{k}^{1}\right)_{\bar{\alpha}}^{*} E_{k}^{*} \mid \widetilde{\alpha}_{A} \in T_{\pi_{A}(\bar{\alpha})}^{*} E^{*}\right. \text { and } \\
\left.\widetilde{\alpha}_{A} \circ{ }_{\pi_{A}(\bar{\alpha})}=\widetilde{\alpha}_{B} \circ{ }_{\pi_{B}(\bar{\alpha})} \text { for all } A \text { and } B\right\}
\end{array}
$$

This implies that the assignment

$$
\bar{\alpha} \in E_{k}^{*} \rightarrow S(\bar{\alpha}) \subseteq\left(T_{k}^{1}\right)_{\bar{\alpha}}^{*} E_{k}^{*}
$$

defines a vector subbundle of $\left(T_{k}^{1}\right)^{*} E_{k}^{*}$ of rank $m k+n$. 
Moreover, in this case, the linear epimorphism

$$
\bar{\Lambda}^{\sharp}: S(\bar{\alpha}) \subseteq\left(T_{k}^{1}\right)_{\bar{\alpha}}^{*} E_{k}^{*} \rightarrow \mathcal{F}(\bar{\alpha}) \subseteq T_{\bar{\alpha}} E_{k}^{*} \subseteq T_{\pi_{1}(\bar{\alpha})} E^{*} \times \ldots \times T_{\pi_{k}(\bar{\alpha})} E^{*}
$$

is given by

$$
\bar{\Lambda}^{\sharp}\left(\left(T_{\bar{\alpha}}^{*} \pi_{1}\right)\left(\widetilde{\alpha}_{1}\right), \ldots,\left(T_{\bar{\alpha}}^{*} \pi_{k}\right)\left(\widetilde{\alpha}_{k}\right)\right)=\left(\Lambda_{E^{*}}^{\sharp}\left(\widetilde{\alpha}_{1}\right), \ldots, \Lambda_{E^{*}}^{\sharp}\left(\widetilde{\alpha}_{k}\right)\right) .
$$

From the above results, it can be concluded that the couple $\left(S, \bar{\Lambda}^{\sharp}\right)$ is a $k$-poly-Poisson structure on $E_{k}^{*}$.

Remark 3.10. If we consider the particular case when $E$ is the standard Lie algebroid $T Q$ then it is clear that $E_{k}^{*}$ is the cotangent bundle of $k$-covelocities $\left(T_{k}^{1}\right)^{*} Q$. In addition, a direct computation proves that the couple $\left(S, \bar{\Lambda}^{\sharp}\right)$ is just the $k$-poly-Poisson structure induced by the $k$ polysymplectic structure on $\left(T_{k}^{1}\right)^{*} Q$ which was described in Example2.7. Note that the fiberwise linear Poisson structure on $T^{*} Q$ induced by the standard Lie algebroid structure on $T Q$ is just the canonical Poisson structure associated to the canonical symplectic structure on $T^{*} Q$ (see Appendix).

On the other hand, if $E$ is a Lie algebra $\mathfrak{g}$, then $E_{k}^{*}$ is just $k$ copies of $\mathfrak{g}^{*}$, the dual of the Lie algebra, which will be denoted by $\mathfrak{g}_{k}^{*}$. Moreover, using the trivialization $T^{*} \mathfrak{g}^{*} \cong \mathfrak{g} \times \mathfrak{g}^{*}$, it is deduced that $\left(T_{k}^{1}\right)^{*} \mathfrak{g}_{k}^{*} \cong \mathfrak{g}_{k}^{*} \times\left(\mathfrak{g}_{k} \times \stackrel{(k}{.} \times \mathfrak{g}_{k}\right), \mathfrak{g}_{k}$ being $k$ copies of $\mathfrak{g}$. Under this identification, given a point $\mu=\left(\mu_{1}, \ldots, \mu_{k}\right) \in \mathfrak{g}_{k}^{*}, S$ is defined by

$$
S(\mu)=\{(\mu,((\xi, 0, \ldots, 0),(0, \xi, \ldots, 0), \ldots,(0,0, \ldots, \xi))) \mid \xi \in \mathfrak{g}\}
$$

and $\bar{\Lambda}^{\sharp}$ is characterized by the following expression

$$
\begin{aligned}
\bar{\Lambda}_{\mu}^{\sharp}(\mu,((\xi, 0, \ldots, 0),(0, \xi, \ldots, 0), \ldots,(0,0, \ldots, \xi))) & =\left(a d_{\xi}^{*} \mu_{1}, a d_{\xi}^{*} \mu_{2}, \ldots, a d_{\xi}^{*} \mu_{k}\right) \\
& =\left(\xi_{\mathfrak{g}^{*}}\left(\mu_{1}\right), \ldots, \xi_{\mathfrak{g}^{*}}\left(\mu_{k}\right)\right) .
\end{aligned}
$$

Therefore, from (2.6), we have that each polysymplectic leaf is a $k$-coadjoint orbit of Example 2.9 .

Another interesting example, which generalizes the previous one, is the Atiyah algebroid of a $G$-principal bundle $Q \rightarrow Q / G=M$. In this case, the Lie algebroid is the vector bundle $T Q / G \rightarrow M$. In section 4 , we will describe the poly-Poisson structure on $T^{*} Q / G \oplus \stackrel{(k}{.} \oplus T^{*} Q / G$ as a consequence of a reduction procedure.

Example 3.11. An example closely related with Example 3.9 is the following one. Given a vector bundle $\tau_{E}: E \rightarrow Q$ of rank $n$ over a manifold $Q$ of dimension $m$, its frame bundle is the bundle $L E \rightarrow M$ whose fiber at $q \in Q$ is the set of linear isomorphisms at $E_{q}$, i.e., $u \in(L E)_{q}$ if $u: \mathbb{R}^{n} \rightarrow E_{q}$ is a linear isomorphism.

The frame bundle is a $G L(n)$-principal bundle. More precisely, if $u \in L E$ and $g \in G L(n)$ then $u \cdot g$ is just the composition. Moreover, we have that $L E$ is an open subset of the Whitney $\operatorname{sum} E_{n}^{*}=E^{*} \oplus \stackrel{(n}{.} \oplus E^{*}$.

Now, if $\tau_{E}: E \rightarrow Q$ is a Lie algebroid with $\Lambda_{E^{*}}$ the fiberwise linear Poisson structure on the dual bundle $E^{*}$ to $E$, then $L E$ is endowed with a poly-Poisson structure. Consider the maps $\pi_{A}: L E \rightarrow E^{*}$ given by

$$
u \in L E \mapsto \pi_{A}(u)=\operatorname{pr}_{A} \circ u^{-1} .
$$

Note that these maps are just the restriction to $L E$ of the canonical projections $\pi_{A}: E_{n}^{*} \rightarrow E^{*}$, $A \in\{1, \ldots, n\}$, of Example 3.9. Thus, taking the foliation $\mathcal{F}$ of the same example restricted to $L E$, one immediately deduce that $L E$ is endowed with a poly-Poisson structure.

Next, it will be considered a different type of example of a poly-Poisson structure, motivated by singular Lagrangian systems. 
Example 3.12 ( $k$-poly-Poisson structures of Dirac type). One of the typical examples of Poisson structures is given by the so-called Poisson structures of Dirac type, which are useful for dealing with singular Lagrangian systems. Let $(M, \omega)$ be a symplectic manifold and let $D$ denote a regular involutive distribution on $M$ such that $D \cap D^{\perp}=0$ where $\perp$ is used to denote the symplectic orthogonal (recall that given a vector subspace $W \subseteq T_{p} M, W^{\perp}=\left(\omega^{b}\right)^{-1}\left(W^{\circ}\right)$ where ${ }^{\circ}$ denotes the annihilator). Then, one may define a Poisson structure $\Lambda_{D}$ on $M$ just by describing the associated bundle map $\Lambda_{D}^{\sharp}$ in the following way:

$$
\Lambda_{D}^{\sharp}(\alpha)=p \circ\left(\omega^{b}\right)^{-1}(\alpha), \quad\left(\alpha \in T^{*} M\right),
$$

$p: T M \rightarrow D$ being the projection over $D$ (note that $T M=D \oplus D^{\perp}$ ). It can be seen that $\Lambda_{D}^{\sharp}$ defined in this way is a Poisson structure. Furthermore, the associated symplectic foliation is just $D$ (for more details see, for instance, [20, 3.8]).

We are going to extend this construction to the poly-Poisson context. Let $\left(\omega_{1}, \ldots, \omega_{k}\right)$ be a $k$-polysymplectic structure on a manifold $M$ of dimension $m$ and $D$ be a regular and involutive distribution of rank $r$ on $M$. In addition, assume that $D$ satisfies

$$
D(p) \cap D^{\perp}(p)=\{0\}, \quad \text { for every } p \in M,
$$

where, in this case, $D^{\perp}$ is defined by

$$
D^{\perp}(p):=\cap_{i=1}^{k}\left(\omega_{i}^{b}\right)^{-1}\left(D^{\circ}(p)\right) .
$$

First, define $S$ as the vector subbundle of $\left(T_{k}^{1}\right)^{*} M$ whose fiber at $p \in M$ is

$$
S(p)=\left\{\bar{\alpha}=\left(\alpha_{1}, \ldots, \alpha_{k}\right) \in\left(T_{k}^{1}\right)_{p}^{*} M \mid \exists X \in D(p) \text { such that } \bar{\alpha}_{\mid D(p)}=\bar{\omega}^{b}(X)_{\mid D(p)}\right\} .
$$

Note that, if $\iota_{D}(p): D(p) \rightarrow T_{p} M$ is the canonical inclusion, then the dimension of the space $\bar{S}(p)=\left[\left(\iota_{D}(p)^{*} \times \ldots \times \iota_{D}(p)^{*}\right) \circ \bar{\omega}^{b}(p)\right](D(p))$ is just $r=\operatorname{dim} D(p)=\operatorname{rank} D$ (this follows using (3.15) $)$. Thus, since

$$
S(p)=\left(\iota_{D}(p)^{*} \times \ldots \times \iota_{D}(p)^{*}\right)^{-1}(\bar{S}(p))
$$

we conclude that the rank of $S$ is $r+(m-r) k$.

Next, the morphism $\bar{\Lambda}^{\sharp}: S \rightarrow T M$ is defined as follows: If $\bar{\alpha} \in S$ then there exists $X \in D$ such that $\bar{\omega}^{b}(X)_{\mid D}=\bar{\alpha}_{\mid D}$. Thus,

$$
\bar{\Lambda}^{\sharp}(\bar{\alpha})=X .
$$

$\bar{\Lambda}^{\sharp}$ is well defined: if $X, Y \in D$ and $\bar{\omega}^{b}(X)_{\mid D}=\bar{\omega}^{b}(Y)_{\mid D}$, then $X-Y \in D^{\perp}$. Therefore, using that $D \cap D^{\perp}=\{0\}$, we get $X=Y$. Let us show that the conditions of Theorem 3.6 hold.

If $\bar{\alpha} \in S$ then there exists $X \in D$ such that $\bar{\omega}^{b}(X)_{\mid D}=\bar{\alpha}_{\mid D}$. Thus,

$$
\bar{\alpha}\left(\bar{\Lambda}^{\sharp}(\bar{\alpha})\right)=\bar{\alpha}(X)=\bar{\omega}^{b}(X)(X)=0,
$$

where, in the last equality, it has been used i) in Proposition 2.3 .

Next, assume that $\bar{\alpha} \in S$ satisfies that $\bar{\alpha}\left(\bar{\Lambda}^{\sharp}(\bar{\beta})\right)=0$ for every $\bar{\beta} \in S$. As a consequence,

$$
\bar{\alpha}(Y)=0 \text {, }
$$

for every $Y \in D$. But, if $\bar{\alpha}_{\mid D}=\bar{\omega}^{b}(X)_{\mid D}$, with $X \in D$, from (3.16) it is deduced that $X \in D \cap D^{\perp}$. Using (3.15), $\bar{\Lambda}^{\sharp}(\bar{\alpha})=X=0$, so ii) in Theorem 3.6 holds.

Finally, a direct computation shows that the generalized distribution

$$
p \in M \rightarrow \mathcal{F}(p)=\bar{\Lambda}^{\sharp}(S(p)) \subseteq T_{p} M,
$$

associated with the pair $\left(S, \bar{\Lambda}^{\sharp}\right)$ is just $D$, which is involutive by hypothesis. Morever, for every leaf $L$, the $\mathbb{R}^{k}$-valued two form $\bar{\omega}_{L}$ is just the pull-back to $L$ of $\bar{\omega}$. $\bar{\omega}_{L}$ is clearly closed and the nondegeneracy (2.1) is a consequence of (3.15).

Thus, using Theorem 3.6. $\left(M, S, \bar{\Lambda}^{\sharp}\right)$ is a poly-Poisson manifold with polysymplectic foliation $D$. 


\section{Poly-Poisson structures And Reduction of POlysymplectic StruCtures}

As it is well known, one can obtain Poisson manifolds from symplectic manifolds through a reduction procedure. Let us recall some details of the process. Let $(M, \omega)$ be a symplectic manifold and $G$ be a Lie group acting freeely and properly on $M$. If the action $\Phi: G \times M \rightarrow M$ preserves the symplectic structure (i.e., $\Phi_{g}^{*} \omega=\omega$, for all $g \in G$ ) then the orbit space $M / G$ inherits a Poisson structure $\Lambda$ in such a way that the bundle projection $\pi: M \rightarrow M / G$ is a Poisson map, that is, $\Lambda^{\sharp}(\pi(x))=T_{x} \pi \circ\left(\omega^{b}(x)\right)^{-1} \circ T_{x}^{*} \pi$, for $x \in M$. A particular example of this situation is the following one. If the action of $G$ on $Q$ is free and proper then it can be lifted to an action on $T^{*} Q$ which preserves the canonical symplectic structure $\omega_{Q}$. The corresponding Poisson structure on $T^{*} Q / G$ is the so-called Poisson-Sternberg structure, and it may be seen as the linear Poisson structure associated to the Atiyah algebroid on $T Q / G$ (see [14]).

We have introduced poly-Poisson structures to play the same role with respect to polysymplectic manifolds, so it is expected that the reduction of a polysymplectic manifold is a poly-Poisson manifold. The next theorem asserts that this is true under certain regularity conditions.

Theorem 4.1. Let $\left(M, \omega_{1}, \ldots, \omega_{k}\right)$ be a polysymplectic manifold and $G$ be a Lie group that acts over $M(\Phi: G \times M \rightarrow M)$ freely, properly and satisfies $\Phi_{g}^{*} \omega_{i}=\omega_{i}$, for every $g \in G$ and $i \in\{1, \ldots, k\}$. Suppose that $V \pi$ is the vertical bundle of the principal bundle projection $\pi: M \rightarrow M / G$ and that the following conditions hold:

i) $\operatorname{Im}\left(\bar{\omega}^{b}\right) \cap(V \pi)^{\circ k}$ is a vector subbundle, where $(V \pi)^{\circ k}=(V \pi)^{\circ} \times \stackrel{(k}{.} \times^{\circ} \times(V \pi)^{\circ}$.

ii) $\left(\bar{\omega}^{b}(p)\right)^{-1}\left(\left(\left(V_{p} \pi\right)^{\perp}\right)^{\circ k} \cap\left(V_{p} \pi\right)^{\circ k} \cap S(p)\right) \subseteq V_{p} \pi$, where $(V \pi)^{\perp}$ is the polysymplectic orthogonal, that is, $(V \pi)^{\perp}=\bigcap_{i=1}^{k}\left(\omega_{i}^{b}\right)^{-1}\left((V \pi)^{\circ}\right)$ and $S$ is the image of $\bar{\omega}^{b}$, that is, $S=\operatorname{Im}\left(\bar{\omega}^{b}\right)$.

Then, there is a natural k-poly-Poisson structure on $M / G$.

Proof. First of all, denote by $\left(T_{p}^{*} \pi\right)_{k}^{1}:\left(T_{k}^{1}\right)_{\pi(p)}^{*}(M / G) \rightarrow\left(T_{k}^{1}\right)_{p}^{*} M$ to be $k$ copies of $T_{p}^{*} \pi$.

From the fact that the 2 -forms $\omega_{1}, \ldots, \omega_{k}$ are $G$-invariant, $S$ is invariant under the lifted action

$$
\begin{aligned}
& \Phi^{\left(T_{k}^{1}\right)^{*}}: \quad G \times\left(T_{k}^{1}\right)^{*} M \quad \rightarrow \quad\left(T_{k}^{1}\right)^{*} M \\
& \left(g,\left(\alpha_{1}, \ldots, \alpha_{k}\right)\right) \rightarrow\left(\Phi_{g^{-1}}^{*} \alpha_{1}, \ldots, \Phi_{g^{-1}}^{*} \alpha_{k}\right) .
\end{aligned}
$$

In addition, $\pi: S \rightarrow M$ is $G$-equivariant.

Now, for every $p \in M$, define the subspace $\hat{S}(\pi(p))$ of $\left(T_{k}^{1}\right)_{\pi(p)}^{*}(M / G)$ given by

$$
\hat{S}(\pi(p)):=\left\{\hat{\alpha}=\left(\alpha_{1}, \ldots, \alpha_{k}\right) \in\left(T_{k}^{1}\right)_{\pi(p)}^{*}(M / G) \mid\left(T_{p}^{*} \pi\right)_{k}^{1}(\hat{\alpha}) \in S(p)\right\} .
$$

Note that the $G$-invariant character of $S$ implies that

$$
\left(T_{g p}^{*} \pi\right)_{k}^{1}(\hat{\alpha}) \in S(g p) \Longleftrightarrow\left(T_{p}^{*} \pi\right)_{k}^{1}(\hat{\alpha}) \in S(p), \quad \forall g \in G .
$$

Thus, the definition of $\hat{S}(\pi(p))$ does not depend on the chosen point $p \in M$.

In fact, since $\left(T_{p}^{*} \pi\right)_{k}^{1}\left(\left(T_{k}^{1}\right)_{\pi(p)}^{*}(M / G)\right)=\left(V_{p} \pi\right)^{\circ k}$, it follows that $\left(T_{p}^{*} \pi\right)_{k}^{1}(\hat{S}(\pi(p)))=S(p) \cap$ $\left(V_{p} \pi\right)^{\circ k}$, for all $p \in M$. In particular, $\hat{S}(\pi(p)) \cong S(p) \cap\left(V_{p} \pi\right)^{\circ k}$.

Now, using hypothesis i) in the theorem, we have that $S \cap(V \pi)^{\circ k}$ is a $G$-invariant vector subbundle of $\left(T_{k}^{1}\right)^{*} M$. Therefore, $\hat{S}$ is isomorphic to the quotient vector subbundle

$$
\left(S \cap(V \pi)^{\circ k}\right) / G=\left(\operatorname{Im}\left(\bar{\omega}^{b}\right) \cap(V \pi)^{\circ k}\right) / G .
$$


Next, define the vector bundle morphism $\hat{\Lambda}^{\sharp}: \hat{S} \rightarrow T(M / G)$ as $\hat{\Lambda}^{\sharp}(\pi(p)):=T_{p} \pi \circ\left(\bar{\omega}^{b}(p)\right)^{-1} \circ$ $\left(T_{p}^{*} \pi\right)_{k \mid \hat{S}(\pi(p))}^{1}$, i.e., $\hat{\Lambda}^{\sharp}(\pi(p))$ makes the following diagram commutative

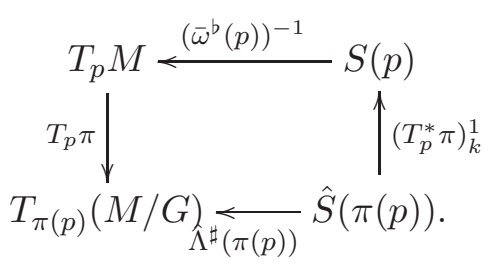

Note that the $G$-invariant character of $\hat{S}$ and of the 2 -forms $\omega_{i}$ implies that the map $\hat{\Lambda}^{\sharp}(\pi(p))$ is well defined (it is independent of the base point $p \in M$ ).

Let us prove that $\left(\hat{S}, \hat{\Lambda}^{\sharp}\right)$ is a poly-Poisson structure on $M / G$.

First, condition i) of Definition 3.1 is a direct consequence of the skew-symmetric character of the monomorphism $\bar{\omega}^{b}$.

In order to prove condition ii) in Definition 3.1, take $\hat{\alpha} \in \hat{S}(\pi(p))$ such that $\hat{\alpha}_{\mid \operatorname{Im}\left(\hat{\Lambda}^{\sharp}(\pi(p))\right)}=0$. From the definition of $\hat{\Lambda}^{\sharp}$ we have

$$
\begin{aligned}
\operatorname{Im}\left(\hat{\Lambda}^{\sharp}(\pi(p))\right) & =\left(T_{p} \pi \circ\left(\bar{\omega}^{b}(p)\right)^{-1} \circ\left(T_{p}^{*} \pi\right)_{k}^{1}\right)(\hat{S}(\pi(p))) \\
& =\left(T_{p} \pi \circ\left(\bar{\omega}^{b}(p)\right)^{-1}\right)\left(S(p) \cap\left(V_{p} \pi\right)^{\circ k}\right)=\left(T_{p} \pi\right)\left(V_{p} \pi\right)^{\perp} .
\end{aligned}
$$

Thus, $\hat{\alpha}_{\mid \operatorname{Im}\left(\hat{\Lambda}^{\sharp}(\pi(p))\right)}=0$ is equivalent to $\left[\left(T_{p}^{*} \pi\right)_{k}^{1}(\hat{\alpha})\right]_{\mid\left(V_{p} \pi\right)^{\perp}}=0$, and, as a consequence, $\hat{\alpha} \in \hat{S}$ with $\hat{\alpha}_{\mid \operatorname{Im}\left(\hat{\Lambda}^{\sharp}(\pi(p))\right)}=0$ if and only if

$$
\left(T_{p}^{*} \pi\right)_{k}^{1}(\hat{\alpha}) \in\left(\left(\left(V_{p} \pi\right)^{\perp}\right)^{\circ k} \cap\left(V_{p} \pi\right)^{\circ k} \cap S(p)\right) .
$$

Therefore, Definition 3.1 ii) is the relation

$$
\left(T_{p} \pi \circ\left(\bar{\omega}^{b}(p)\right)^{-1}\right)\left(\left(\left(V_{p} \pi\right)^{\perp}\right)^{\circ k} \cap\left(V_{p} \pi\right)^{\circ k} \cap S(p)\right)=0,
$$

and using that $V_{p} \pi=\operatorname{Ker}\left(T_{p} \pi\right)$, this is equivalent to

$$
\left(\bar{\omega}^{b}(p)\right)^{-1}\left(\left(\left(V_{p} \pi\right)^{\perp}\right)^{\circ k} \cap\left(V_{p} \pi\right)^{\circ k} \cap S(p)\right) \subseteq V_{p} \pi,
$$

which is just the second hypothesis in the theorem.

Finally, we will prove that the integrability condition (3.1) holds for the couple $\left(\hat{S}, \hat{\Lambda}^{\sharp}\right)$.

Denote by $\Lambda^{\sharp}: S \subseteq\left(T_{k}^{1}\right)^{*} M \rightarrow T M$ the isomorphism $\left(\bar{\omega}^{b}\right)^{-1}$ and by

$$
\left(\pi_{k}^{1}\right)^{*}: \Gamma\left(\left(T_{k}^{1}\right)^{*}(M / G)\right) \rightarrow \Gamma\left(\left(T_{k}^{1}\right)^{*} M\right)
$$

the monomorphism of $C^{\infty}(M / G)$-modules induced by the projection $\pi$ between the spaces $\Gamma\left(\left(T_{k}^{1}\right)^{*}(M / G)\right)$ and $\Gamma\left(\left(T_{k}^{1}\right)^{*} M\right)$ of sections of the vector bundles $\left(T_{k}^{1}\right)^{*}(M / G) \rightarrow M / G$ and $\left(T_{k}^{1}\right)^{*} M \rightarrow M$, respectively. Then, if $\hat{\alpha}, \hat{\beta} \in \Gamma\left(\left(T_{k}^{1}\right)^{*}(M / G)\right)$ it follows that the vector fields $\Lambda^{\sharp}\left(\left(\pi_{k}^{1}\right)^{*} \hat{\alpha}\right)$ and $\Lambda^{\sharp}\left(\left(\pi_{k}^{1}\right)^{*} \hat{\beta}\right)$ are $\pi$-projectable on the vector fields $\hat{\Lambda}^{\sharp}(\hat{\alpha})$ and $\hat{\Lambda}^{\sharp}(\hat{\beta})$, respectively. Moreover, since the couple $\left(S, \Lambda^{\sharp}\right)$ is a poly-Poisson structure on $M$, we deduce that

$$
\left[\Lambda^{\sharp}\left(\left(\pi_{k}^{1}\right)^{*} \hat{\alpha}\right), \Lambda^{\sharp}\left(\left(\pi_{k}^{1}\right)^{*} \hat{\beta}\right)\right]=\left(\Lambda^{\sharp} \circ\left(\pi_{k}^{1}\right)^{*}\right)\left(\mathcal{L}_{\hat{\Lambda}^{\sharp}(\hat{\alpha})} \hat{\beta}-\mathcal{L}_{\hat{\Lambda}^{\sharp}(\hat{\beta})} \hat{\alpha}-\mathrm{d}\left(\hat{\beta}\left(\hat{\Lambda}^{\sharp}(\hat{\alpha})\right)\right)\right) .
$$

Thus, if we project on $M / G$ using $\pi$, we conclude that

$$
\left[\hat{\Lambda}^{\sharp}(\hat{\alpha}), \hat{\Lambda}^{\sharp}(\hat{\beta})\right]=\hat{\Lambda}^{\sharp}\left(\mathcal{L}_{\hat{\Lambda}^{\sharp}(\hat{\alpha})} \hat{\beta}-\mathcal{L}_{\hat{\Lambda}^{\sharp}(\hat{\beta})} \hat{\alpha}-\mathrm{d}\left(\hat{\beta}\left(\hat{\Lambda}^{\sharp}(\hat{\alpha})\right)\right)\right) .
$$


Example 4.2 (Reduction of the cotangent bundle of $k$-covelocities associated with a Lie group). Let $G$ be a Lie group. The cotangent bundle of $k$-covelocities of $G,\left(T_{k}^{1}\right)^{*} G$ has a canonical $k$ polysymplectic structure (Example 2.7). In addition, the action of $G$ on itself by left translations

$$
\begin{aligned}
L: \quad G \times G & \longrightarrow G \\
(g, h) & \rightarrow L(g, h)=g \cdot h
\end{aligned}
$$

can be lifted to an action $L^{\left(T_{k}^{1}\right)^{*}}$ on $\left(T_{k}^{1}\right)^{*} G$ by

$$
\begin{array}{ccc}
L^{\left(T_{k}^{1}\right)^{*}:} \begin{array}{c}
G \times\left(T_{k}^{1}\right)^{*} G \\
\left(g,\left(\alpha_{1}, \ldots, \alpha_{k}\right)\right)
\end{array} \quad \rightarrow \quad\left(T_{k}^{1}\right)^{*} G \\
& \rightarrow\left(L_{g^{-1}}^{*} \alpha_{1}, \ldots, L_{g^{-1}}^{*} \alpha_{k}\right) .
\end{array}
$$

If we consider the left trivialization of the tangent bundle to $G, T G \cong G \times \mathfrak{g}$, and the corresponding trivialization $\left(T_{k}^{1}\right)^{*} G \cong G \times \mathfrak{g}_{k}^{*}, \mathfrak{g}_{k}^{*}=\mathfrak{g}^{*} \times \stackrel{(k}{*} \times \mathfrak{g}^{*}$, the action (4.3) can be written as

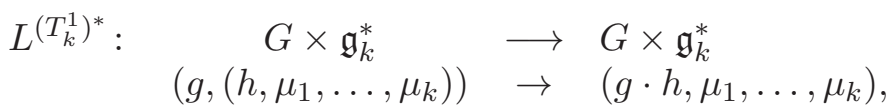

and the associated principal bundle $\pi:\left(T_{k}^{1}\right)^{*} G \rightarrow\left(\left(T_{k}^{1}\right)^{*} G\right) / G$ can be identified with the trivial principal bundle $\pi: G \times \mathfrak{g}_{k}^{*} \rightarrow \mathfrak{g}_{k}^{*}$. Thus, the vertical bundle takes the expression

$$
V \pi_{\left(g, \mu_{1}, \ldots, \mu_{k}\right)}=\left\{(X, 0, \ldots, 0) \text { such that } X \in T_{g} G\right\} .
$$

Therefore, its annihilator is

$$
(V \pi)_{\left(g, \mu_{1}, \ldots, \mu_{k}\right)}^{\circ}=\left\{\left(0, \xi_{1}, \xi_{2}, \ldots, \xi_{k}\right) \mid \xi_{1}, \ldots, \xi_{k} \in \mathfrak{g}\right\} .
$$

We will show that the action of $G$ on $\left(T_{k}^{1}\right)^{*} G \cong G \times \mathfrak{g}_{k}^{*}$, endowed with the canonical polysymplectic structure, satisfies the hypotheses of Theorem 4.1.

First of all we are going to compute explicitly the polysymplectic structure on $G \times \mathfrak{g}_{k}^{*}$. For this purpose, it is first described the canonical symplectic structure $\omega$ on $T^{*} G \cong G \times \mathfrak{g}^{*}$ (see for instance, [19]). Using the left translation by $g \in G$ we can identify $T_{g} G$ and $T_{g}^{*} G$ with $\mathfrak{g}$ and $\mathfrak{g}^{*}$, respectively. Thus, we have that $T_{(g, \mu)}\left(G \times \mathfrak{g}^{*}\right) \cong \mathfrak{g} \times \mathfrak{g}^{*}$ and $T_{(g, \mu)}^{*}\left(G \times \mathfrak{g}^{*}\right) \cong \mathfrak{g}^{*} \times \mathfrak{g}$ and, under these identifications, it follows that

$$
\begin{aligned}
& \omega_{(g, \mu)}: T_{(g, \mu)}\left(G \times \mathfrak{g}^{*}\right) \times T_{(g, \mu)}\left(G \times \mathfrak{g}^{*}\right) \cong\left(\mathfrak{g} \times \mathfrak{g}^{*}\right) \times\left(\mathfrak{g} \times \mathfrak{g}^{*}\right) \longrightarrow \mathbb{R} \\
& \left(\left(\xi_{1}, \tau_{1}\right),\left(\xi_{2}, \tau_{2}\right)\right) \quad \rightarrow \omega_{(g, \mu)}\left(\left(\xi_{1}, \tau_{1}\right),\left(\xi_{2}, \tau_{2}\right)\right) \\
& =\tau_{2}\left(\xi_{1}\right)-\tau_{1}\left(\xi_{2}\right)+\mu\left(\left[\xi_{1}, \xi_{2}\right]\right) \text {. }
\end{aligned}
$$

Therefore,

$$
\begin{aligned}
& \omega_{(g, \mu)}^{b}: T_{(g, \mu)}\left(G \times \mathfrak{g}^{*}\right) \cong \mathfrak{g} \times \mathfrak{g}^{*} \longrightarrow T_{(g, \mu)}^{*}\left(G \times \mathfrak{g}^{*}\right) \cong \mathfrak{g}^{*} \times \mathfrak{g} \\
& (\xi, \tau) \quad \rightarrow \quad \omega_{(g, \mu)}^{b}(\xi, \tau)=\left(a d_{\xi}^{*} \mu-\tau, \xi\right),
\end{aligned}
$$

and, as a consequence, $\left(\omega_{(g, \mu)}^{b}\right)^{-1}(\tau, \xi)=\left(\xi, a d_{\xi}^{*} \mu-\tau\right)$, for $(\tau, \xi) \in \mathfrak{g}^{*} \times \mathfrak{g} \cong T_{(g, \mu)}^{*}\left(G \times \mathfrak{g}^{*}\right)$.

Now, denote by $\bar{\omega}^{b}: T\left(\left(T_{k}^{1}\right)^{*} G\right) \rightarrow\left(T_{k}^{1}\right)^{*}\left(\left(T_{k}^{1}\right)^{*} G\right)$ the vector bundle morphism associated with the polysymplectic structure on $\left(T_{k}^{1}\right)^{*} G \cong G \times \mathfrak{g}_{k}^{*}$. Then, from Eq. (4.8) and definition of the canonical $k$-polysymplectic structure on $\left(T_{k}^{1}\right)^{*} G$ we have

$$
\begin{aligned}
\bar{\omega}_{\left(g, \mu_{1}, \ldots, \mu_{k}\right)}^{b}\left(\xi, \tau_{1}, \ldots, \tau_{k}\right)= & \left(\left(a d_{\xi}^{*} \mu_{1}-\tau_{1}, \xi, 0, \ldots, 0\right),\left(a d_{\xi}^{*} \mu_{2}-\tau_{2}, 0, \xi, 0, \ldots, 0\right), \ldots,\right. \\
& \left., \ldots,\left(a d_{\xi}^{*} \mu_{k}-\tau_{k}, 0,0, \ldots, \xi\right)\right),
\end{aligned}
$$

for $\left(\xi, \tau_{1}, \ldots, \tau_{k}\right) \in \mathfrak{g} \times \mathfrak{g}_{k}^{*}$. In addition, using Eq. (4.9), it is deduced that

$$
\left(S \cap(V \pi)^{\circ k}\right)_{\left(g, \mu_{1}, \ldots, \mu_{k}\right)}=\{((0, \xi, 0, \ldots, 0),(0,0, \xi, 0, \ldots, 0), \ldots,(0,0,0, \ldots, \xi)) \mid \xi \in \mathfrak{g}\}
$$

which implies that hypothesis i) in Theorem 4.1 holds. 
On the other hand, from Eq. (4.8) and (4.9), we obtain

$$
(V \pi)_{\left(g, \mu_{1}, \ldots, \mu_{k}\right)}^{\perp}=\left\{\left(\xi, a d_{\xi}^{*} \mu_{1}, a d_{\xi}^{*} \mu_{2}, \ldots, a d_{\xi}^{*} \mu_{k}\right) \mid \xi \in \mathfrak{g}\right\}
$$

and, as a consequence,

$$
\left((V \pi) \stackrel{\perp}{\left(g, \mu_{1}, \ldots, \mu_{k}\right)}\right)^{\circ}=\left\{\left(a d_{\xi_{1}}^{*} \mu_{1}+\ldots+a d_{\xi_{k}}^{*} \mu_{k}, \xi_{1}, \xi_{2}, \ldots, \xi_{k}\right) \mid \xi_{i} \in \mathfrak{g}, i \in\{1, \ldots, k\}\right\} .
$$

Therefore, we deduce that $\left.\left((V \pi)^{\perp^{\circ}}\right)^{k} \cap(V \pi)^{\circ k} \cap \operatorname{Im}\left(\bar{\omega}^{b}\right)\right)_{\left(g, \mu_{1}, \ldots, \mu_{k}\right)}$ is just

$$
\left\{((0, \eta, 0, \ldots, 0),(0,0, \eta, \ldots, 0), \ldots,(0,0,0, \ldots, \eta)) \mid \eta \in \mathfrak{g}, a d_{\eta}^{*} \mu_{i}=0, i \in\{1, \ldots, k\}\right\} .
$$

Thus, given an element of $\left.\left((V \pi)^{\perp^{\circ}}\right)^{k} \cap(V \pi)^{\circ k} \cap \operatorname{Im}\left(\bar{\omega}^{b}\right)\right)_{\left(g, \mu_{1}, \ldots, \mu_{k}\right)}$,

$$
\begin{aligned}
\left(\bar{\omega}^{b}\right)_{\left(g, \mu_{1}, \ldots, \mu_{k}\right)}^{-1}((0, \eta, 0, \ldots, 0),(0,0, \eta, \ldots, 0), \ldots,(0,0,0, \ldots, \eta)) & =\left(\eta, a d_{\eta}^{*} \mu_{1}, a d_{\eta}^{*} \mu_{2}, \ldots, a d_{\eta}^{*} \mu_{k}\right) \\
& =(\eta, 0,0, \ldots, 0)
\end{aligned}
$$

which implies that this vector is vertical. So, the hypothesis ii) of Theorem 4.1 is satisfied.

Now, let us give an explicit description of $\hat{S}$ and $\bar{\Lambda}^{\sharp}$.

Computation of $\hat{S}$ : Recall by Theorem 4.1, that $\hat{S}$ is the subbundle of $\mathfrak{g}_{k}^{*}$ given by $\hat{S} \cong$ $\left(S \cap(V \pi)^{\circ k}\right) / G=\left(\operatorname{Im}\left(\bar{\omega}^{b}\right) \cap(V \pi)^{\circ k}\right) / G$. By (4.10) and the definition of the action (4.4) is obvious that the fiber of $\hat{S}$ at a point $\mu=\left(\mu_{1}, \ldots, \mu_{k}\right) \in \mathfrak{g}_{k}^{*}$ is given by

$$
\hat{S}_{\mu}=\{((\xi, 0, \ldots, 0),(0, \xi, \ldots, 0), \ldots,(0,0, \ldots, \xi)) \mid \xi \in \mathfrak{g}\}
$$

Computation of $\hat{\Lambda}^{\sharp}$ : Remember that $\hat{\Lambda}^{\sharp}$ is given by $T_{(g, \mu)} \pi \circ\left(\bar{\omega}_{(g, \mu)}^{b}\right)^{-1} \circ\left(T_{(g, \mu)}^{*} \pi\right)_{k}^{1}$ So on $\mu=\left(\mu_{1}, \ldots, \mu_{k}\right) \in \mathfrak{g}_{k}^{*}$, given $((\xi, 0, \ldots, 0),(0, \xi, \ldots, 0), \ldots,(0,0, \ldots, \xi)) \in \hat{S}_{\mu}$

$$
\begin{aligned}
& \hat{\Lambda}_{\mu}^{\sharp}((\xi, 0, \ldots, 0),(0, \xi, \ldots, 0), \ldots,(0,0, \ldots, \xi)) \\
& =\left(T_{(g, \mu)} \pi \circ\left(\bar{\omega}_{(g, \mu)}^{b}\right)^{-1} \circ\left(T_{(g, \mu)}^{*} \pi\right)_{k}^{1}\right)((\xi, 0, \ldots, 0),(0, \xi, \ldots, 0), \ldots,(0,0, \ldots, \xi)) \\
& \left.=\left(T_{(g, \mu)} \pi \circ\left(\bar{\omega}_{(g, \mu)}^{b}\right)^{-1}\right)((0, \xi, 0, \ldots, 0),(0,0, \xi, \ldots, 0), \ldots,(0,0,0, \ldots, \xi))\right) \\
& =T_{(g, \mu)} \pi\left(\xi, a d_{\xi}^{*} \mu_{1}, a d_{\xi}^{*} \mu_{2}, \ldots, a d_{\xi}^{*} \mu_{k}\right) \\
& =\left(a d_{\xi}^{*} \mu_{1}, a d_{\xi}^{*} \mu_{2}, \ldots, a d_{\xi}^{*} \mu_{k}\right)
\end{aligned}
$$

where we used (4.9).

Note that this poly-Poisson structure coincides with the one obtained using the construction of Example 3.9 (see (3.12) and (3.13) in Remark 3.10). Moreover, as a consequence, we have that the polysymplectic leaves are just the orbits of the $k$-coadjoint action.

Example 4.3 (Reduction of the cotangent bundle of $k$-covelocitites associated with the total space of a principal bundle). Let $Q$ be a manifold of dimension $n$ endowed with a free and proper action of a Lie group $G, \Psi: G \times Q \rightarrow Q$. Let $\tilde{\pi}: Q \rightarrow Q / G$ be the corresponding principal bundle projection. As we did in the previous example, we can define the lifted action

$$
\begin{aligned}
& \Psi^{\left(T_{k}^{1}\right)^{*}:} \quad G \times\left(T_{k}^{1}\right)^{*} Q \quad \longrightarrow\left(T_{k}^{1}\right)^{*} Q \\
& \left(g,\left(\alpha_{1}, \ldots, \alpha_{k}\right)\right) \rightarrow\left(\Psi_{g^{-1}}^{*} \alpha_{1}, \ldots, \Psi_{g^{-1}}^{*} \alpha_{k}\right)
\end{aligned}
$$

and this action still is free and proper. Thus, we have the principal bundle $\pi:\left(T_{k}^{1}\right)^{*} Q \rightarrow$ $\left(\left(T_{k}^{1}\right)^{*} Q\right) / G$ and, moreover, $\left(\left(T_{k}^{1}\right)^{*} Q\right) / G \cong T^{*} Q / G \oplus \stackrel{(k}{.} \oplus T^{*} Q / G$.

Let us see that the action of $G$ on the polysymplectic manifold $\left(T_{k}^{1}\right)^{*} Q$ satisfies the conditions of Theorem 4.1. It is clear that the lifted action preserves the polysymplectic structure. In fact, since the lifted action of $G$ on $T^{*} Q$ preserves the canonical symplectic structure $\omega_{Q}$, the action $\Psi^{\left(T_{k}^{1}\right)^{*}}$ preserves the polysymplectic structure on $\left(T_{k}^{1}\right)^{*} Q$. 
Next, let $U$ be an open subset of $Q / G$ such that $\tilde{\pi}^{-1}(U)$ is a trivializing open subset of $Q$, that is $\tilde{\pi}^{-1}(U) \cong U \times G$ and the principal action of $G$ on $\tilde{\pi}^{-1}(U) \cong U \times G$ is given by $\Psi(g,(u, h))=(u, g h)$, for $g \in G$ and $(u, h) \in U \times G$. As a consequence, $\left(T_{k}^{1}\right)^{*}\left(\tilde{\pi}^{-1}(U)\right) \cong$ $\left(T_{k}^{1}\right)^{*} U \times\left(T_{k}^{1}\right)^{*} G$. Moreover, if $\bar{\omega}_{U}$ and $\bar{\omega}_{G}$ are the canonical polysymplectic forms on $\left(T_{k}^{1}\right)^{*} U$ and $\left(T_{k}^{1}\right)^{*} G$ respectively then the polysymplectic form on $\left(T_{k}^{1}\right)^{*}\left(\tilde{\pi}^{-1}(U)\right) \cong\left(T_{k}^{1}\right)^{*}(U) \times\left(T_{k}^{1}\right)^{*} G$, is given by $\bar{\omega}_{U} \times \bar{\omega}_{G}$.

On the other hand, under the identification $\left(T_{k}^{1}\right)^{*}\left(\tilde{\pi}^{-1}(U)\right) \cong\left(T_{k}^{1}\right)^{*}(U) \times\left(T_{k}^{1}\right)^{*} G$ the lifted action of $G$ on $\left(T_{k}^{1}\right)^{*}\left(\tilde{\pi}^{-1}(U)\right)$ is given by

$$
\Psi^{\left(T_{k}^{1}\right)^{*}}(g,(\widetilde{\alpha}, \widetilde{\gamma}))=\left(\widetilde{\alpha}, L^{\left(T_{k}^{1}\right)^{*}}(g, \widetilde{\gamma})\right)
$$

for $g \in G$ and $(\widetilde{\alpha}, \widetilde{\gamma}) \in\left(T_{k}^{1}\right)^{*}(U) \times\left(T_{k}^{1}\right)^{*} G$. Therefore, the quotient space $\left(T_{k}^{1}\right)^{*}\left(\tilde{\pi}^{-1}(U)\right) / G$ is just $\left(T_{k}^{1}\right)^{*}(U) \times \mathfrak{g}_{k}^{*}$, where it has been used the identification $\left(T_{k}^{1}\right)^{*} G \cong G \times \mathfrak{g}_{k}^{*}$ as in Example 4.2

Now, we will prove that the polysymplectic structure on $\left(T_{k}^{1}\right)^{*}\left(\tilde{\pi}^{-1}(U)\right) \cong\left(T_{k}^{1}\right)^{*}(U) \times\left(T_{k}^{1}\right)^{*} G \cong$ $\left(T_{k}^{1}\right)^{*} U \times\left(G \times \mathfrak{g}_{k}^{*}\right)$ and the action of $G$ on $\left(T_{k}^{1}\right)^{*}\left(\tilde{\pi}^{-1}(U)\right)$ given by (4.12), satisfy the hypotheses of Theorem 4.1 .

In order to check these hypotheses, we split the problem in two parts, the side of $\left(T_{k}^{1}\right)^{*} U$ and the one of $\left(T_{k}^{1}\right)^{*} G$. Using that the intersection of the vertical bundle to $\pi$ with $T\left(\left(T_{k}^{1}\right)^{*} U\right)$ is trivial and the results in the previous Example 4.2, we deduce that the hypotheses of Theorem 4.1 hold.

On the other hand, from (4.1), it follows that:

$$
\hat{S}(\pi(\bar{\alpha}))=\left\{\left(\hat{\alpha}_{1}, \ldots, \hat{\alpha}_{k}\right) \in\left(T_{k}^{1}\right)_{\pi(\bar{\alpha})}^{*}\left(T^{*} Q / G \oplus \stackrel{(k}{*} \oplus T^{*} Q / G\right) \mid\left(T_{\bar{\alpha}}^{*} \pi\right)_{k}^{1}\left(\hat{\alpha}_{1}, \ldots, \hat{\alpha}_{k}\right) \in S(\bar{\alpha})\right\},
$$

for $\bar{\alpha} \in\left(T_{k}^{1}\right)_{q}^{*} Q$, where $S=\operatorname{Im}\left(\bar{\omega}^{b}\right)$ and $\bar{\omega}$ is the canonical polysymplectic structure on $\left(T_{k}^{1}\right)^{*} Q$.

This implies that $\hat{S}$ is given by (3.11). Thus, $\left(\hat{\alpha}_{1}, \ldots, \hat{\alpha}_{k}\right) \in \hat{S}(\pi(\bar{\alpha}))$ if

$$
\hat{\alpha}_{A}=\left(T_{\bar{\alpha}}^{*} \tilde{\pi}_{A}\right)\left(\tilde{\alpha}_{A}\right) \text {, with } \tilde{\alpha}_{A} \in T_{\tilde{\pi}_{A}(\bar{\alpha})}^{*}\left(T^{*} Q / G\right)
$$

and

$$
\tilde{\alpha}_{A} \circ \stackrel{\mathrm{\pi}}{\mathrm{\pi}}_{A}^{\mathrm{v}(\bar{\alpha})}=\tilde{\alpha}_{B} \circ \stackrel{\mathrm{\pi}}{\mathrm{\pi}}(\bar{\alpha})^{\mathrm{v}}, \text { for all } A \text { and } B,
$$

$\tilde{\pi}_{A}: T^{*} Q / G \oplus \stackrel{(k}{.} \oplus T^{*} Q / G \rightarrow T^{*} Q / G$ being the canonical projection over the $A$ th-factor.

In order to compute $\hat{\Lambda}^{\sharp}$, we have to take into account that, from Theorem 4.1, $\hat{\Lambda}_{\tilde{\pi}\left(\widetilde{\alpha}_{1}, \ldots, \widetilde{\alpha}_{k}\right)}^{\sharp}=$ $T_{\left(\alpha_{1}, \ldots, \alpha_{k}\right)} \pi \circ\left(\bar{\omega}_{\left(\alpha_{1}, \ldots, \alpha_{k}\right)}^{b}\right)^{-1} \circ\left(T_{\left(\alpha_{1}, \ldots, \alpha_{k}\right)}^{*} \pi\right)_{k}^{1}$. Moreover, if $\tilde{\alpha}_{A} \in T_{\tilde{\pi}_{A}(\bar{\alpha})}^{*}\left(T^{*} Q / G\right)$, for all $A$, and

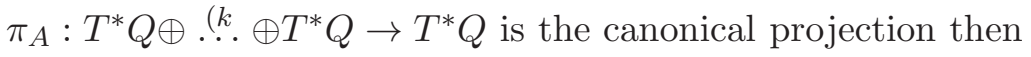

$$
\hat{\Lambda}_{\pi(\bar{\alpha})}^{\sharp}\left(\left(T_{\bar{\alpha}}^{*} \pi_{1}\right)\left(\tilde{\alpha}_{1}\right), \ldots,\left(T_{\bar{\alpha}}^{*} \pi_{k}\right)\left(\tilde{\alpha}_{k}\right)\right)=\left(T_{\bar{\alpha}} \pi \circ\left(\bar{\omega}_{\bar{\alpha}}^{b}\right)^{-1} \circ T_{\bar{\alpha}}^{*} \pi\right)\left(\left(T_{\bar{\alpha}}^{*} \pi_{1}\right)\left(\tilde{\alpha}_{1}\right), \ldots,\left(T_{\bar{\alpha}}^{*} \pi_{k}\right)\left(\tilde{\alpha}_{k}\right)\right) .
$$

The poly-Poisson structure on $T^{*} Q / G \oplus \ldots \oplus T^{*} Q / G$ is just the one associated to the linear Poisson structure on $T^{*} Q / G$ associated to the Atiyah algebroid $T Q / G$ (see Example 3.9).

Remark 4.4. Let $Q$ be an arbitrary manifold of dimension $n$. Then, the frame bundle $\pi: L Q \rightarrow Q$ is endowed with a polysymplectic structure (see Example 2.4) and, moreover, it is an open subset of $\left(T_{n}^{1}\right)^{*} Q$, and the restriction of the polysymplectic structure on $\left(T_{n}^{1}\right)^{*} Q$ is just $-\mathrm{d} \vartheta$ (see Remark 2.8).

If $G$ is a Lie group acting freely and properly on $Q$ then $G$ acts on $\left(T_{n}^{1}\right)^{*} Q$. In addition, $L Q$ is stable under this lifted action (note that, since $\Psi_{g}: Q \rightarrow Q$ is diffeomorphism for any $g \in G$, $T \Psi_{g}: T_{q} Q \rightarrow T_{\Psi_{g}(q)} Q$ is an isomorphism). Thus, from Theorem 4.1, the quotient space $L Q / G$ is a poly-Poisson structure. But a simple computation shows that $L Q / G$ is just the set

$$
\left\{u: \mathbb{R}^{n} \rightarrow(T Q / G)_{[q]} \text { linear isomorphism } \mid[q] \in Q / G\right\},
$$


that is, $L Q / G$ is $L(T Q / G)$, the frame bundle associated to the Atiyah algebroid $T Q / G$. The computations in Example 4.3 allow to conclude that the poly-Poisson structure on $L Q / G$ obtained from the reduction procedure is the poly-Poisson structure on $L(T Q / G)$ from Example 3.11

\section{Appendix: Lie Algebroids And fiberWise Linear Poisson structures}

We will review some basic facts on Lie algebroids and fiberwise linear Poisson structures (for more, details, see [14]).

A Lie algebroid is a real vector bundle $\tau_{E}: E \rightarrow Q$ of rank $n$ over a manifold $Q$ of dimension $m$ such that the space of sections $\Gamma(E)$ admits a Lie algebra structure $\llbracket \cdot, \cdot \rrbracket_{E}$ and, moreover, there exists a vector bundle map $\rho_{E}: E \rightarrow T Q$, the anchor map, such that if we also denote by $\rho_{E}: \Gamma(E) \rightarrow \mathfrak{X}(Q)$ the corresponding morphism of $C^{\infty}(Q)$-modules then

$$
\llbracket X, f Y \rrbracket_{E}=f \llbracket X, Y \rrbracket_{E}+\rho_{E}(X)(f) Y,
$$

for $X, Y \in \Gamma(E)$ and $f \in C^{\infty}(Q)$.

The previous conditions imply that $\rho_{E}$ is a Lie algebra morphism, that is,

$$
\rho_{E} \llbracket X, Y \rrbracket_{E}=\left[\rho_{E}(X), \rho_{E}(Y)\right], \quad \text { for } X, Y \in \Gamma(E) .
$$

Some examples of Lie algebroids are the tangent bundle to a manifold $Q$ and a real Lie algebra of finite dimension.

Let $\left(\llbracket \cdot, \cdot \rrbracket_{E}, \rho_{E}\right)$ be a Lie algebroid structure on a real vector bundle $\tau_{E}: E \rightarrow Q$. If $\left(q^{i}\right)$ are local coordinates on an open subset $U \subseteq Q$ and $\left\{e_{\alpha}\right\}$ is a local basis of $\Gamma(E)$ then

$$
\rho_{E}\left(e_{\alpha}\right)=\rho_{\alpha}^{i} \frac{\partial}{\partial q^{i}}, \quad \llbracket e_{\alpha}, e_{\beta} \rrbracket_{E}=\mathcal{C}_{\alpha \beta}^{\gamma} e_{\gamma},
$$

with $\rho_{\alpha}^{i}, \mathrm{e}_{\alpha \beta}^{\gamma} \in C^{\infty}(U)$ the local structure functions of $E$ with respect to the local coordinates $\left(q^{i}\right)$ and the local basis $\left\{e_{\alpha}\right\}$.

The local structure functions satisfy the the local structure equations on $E$

$$
\rho_{\alpha}^{i} \frac{\partial \rho_{\beta}^{j}}{\partial q^{i}}-\rho_{\beta}^{i} \frac{\partial \rho_{\alpha}^{j}}{\partial q^{i}}=\rho_{\gamma}^{j} \mathfrak{C}_{\alpha \beta}^{\gamma}, \quad \sum_{\operatorname{cyclic}(\alpha, \beta, \gamma)}\left(\rho_{\alpha}^{i} \frac{\partial \mathcal{C}_{\beta \gamma}^{\nu}}{\partial q^{i}}+\mathcal{C}_{\alpha \mu}^{\nu} \mathcal{e}_{\beta \gamma}^{\mu}\right)=0 .
$$

Let $\tau_{E^{*}}: E^{*} \rightarrow Q$ be the dual bundle to a Lie algebroid $\tau_{E}: E \rightarrow Q$. Then, $E^{*}$ admits a fiberwise linear Poisson bracket $\{\cdot, \cdot\}_{E^{*}}$ which is characterized by the following relations,

$$
\begin{gathered}
\{\widehat{X}, \widehat{Y}\}_{E^{*}}=-\llbracket{\widehat{X, Y \rrbracket_{E}}}, \quad\left\{f \circ \tau_{E^{*}}, \widehat{Y}\right\}_{E^{*}}=\rho_{E}(Y)(f) \circ \tau_{E^{*}}, \\
\left\{f \circ \tau_{E^{*}}, g \circ \tau_{E^{*}}\right\}_{E^{*}}=0,
\end{gathered}
$$

for $X, Y \in \Gamma(E)$ and $f, g \in C^{\infty}(Q)$. Here, if $Z \in \Gamma(E)$ we will denote by $\widehat{Z}: E^{*} \rightarrow \mathbb{R}$ the corresponding fiberwise linear function on $E^{*}$.

Let $\left(q^{i}\right)$ be local coordinates on an open subset $U \subseteq Q$ and $\left\{e_{\alpha}\right\}$ be a local basis of $\Gamma\left(\tau_{E}^{-1}(U)\right)$. Denote by $\left(q^{i}, p_{\alpha}\right)$ the corresponding local coordinates on $E^{*}$. Thus,

$$
\left\{p_{\alpha}, p_{\beta}\right\}_{E^{*}}=-\mathrm{C}_{\alpha \beta}^{\gamma} p_{\gamma},\left\{q^{i}, p_{\alpha}\right\}_{E^{*}}=\rho_{\alpha}^{i},\left\{q^{i}, q^{j}\right\}_{E^{*}}=0 .
$$

Therefore, if $\Lambda_{E^{*}}$ is the corresponding Poisson 2-vector on $E^{*}$, it follows that

$$
\Lambda_{E^{*}}^{\sharp}\left(\mathrm{d} q^{i}\right)=\rho_{\alpha}^{i} \frac{\partial}{\partial p_{\alpha}}, \Lambda_{E^{*}}^{\sharp}\left(\mathrm{d} p_{\alpha}\right)=-\left(\rho_{\alpha}^{i} \frac{\partial}{\partial q^{i}}+\mathcal{C}_{\alpha \beta}^{\gamma} p_{\gamma} \frac{\partial}{\partial p_{\beta}}\right) .
$$

Remark A.5. $\quad$ i) The fiberwise linear Poisson structure on $T^{*} Q$ induced by the standard Lie algebroid structure on $T Q$ is just the canonical Poisson structure on $T^{*} Q$ induced by the canonical symplectic structure on $T^{*} Q$. 
ii) If $\mathfrak{g}$ is a real Lie algebra of finite dimension then the linear Poisson structure on $\mathfrak{g}^{*}$ is just the Lie-Poisson structure.

Remark A.6. Suppose that $\alpha_{A} \in E_{q}^{*}$ and $\widetilde{\alpha}_{A} \in T_{\alpha_{A}}^{*} E^{*}$, with $A \in\{1,2\}$ and $q \in Q$. Using (A.2), we deduce that

$$
\left(T_{\alpha_{1}} \tau_{E^{*}}\right)\left(\Lambda_{E^{*}}^{\sharp}\left(\widetilde{\alpha}_{1}\right)\right)=\left(T_{\alpha_{2}} \tau_{E^{*}}\right)\left(\Lambda_{E^{*}}^{\sharp}\left(\widetilde{\alpha}_{2}\right)\right) \Longleftrightarrow \widetilde{\alpha}_{1} \circ \underset{\alpha_{1}}{\mathrm{v}} \circ \rho_{E \mid T_{q}^{*} Q}^{*}=\widetilde{\alpha}_{2} \circ \stackrel{\mathrm{v}}{\alpha_{2}} \circ \rho_{E \mid T_{q}^{*} Q}^{*},
$$

where $\rho_{E}^{*}: T^{*} Q \rightarrow E^{*}$ is the dual morphism of the anchor map $\rho_{E}: E \rightarrow T Q$ and ${ }_{\alpha_{A}}^{\mathrm{v}}: E_{q}^{*} \rightarrow$ $T_{\alpha_{A}} E_{q}^{*}$ is the canonical isomorphism between $E_{q}^{*}$ and $T_{\alpha_{A}} E_{q}^{*}$. We remark that

$$
\mathrm{\alpha}_{A}{ }^{\mathrm{v}}\left(e_{\beta}(q)\right)=\frac{\partial}{\partial p_{\beta} \mid \alpha_{A}}, \quad \text { for } \beta \in\{1, \ldots, n\}
$$

Acknowledgments This work has been partially supported by MICINN (Spain) Grants MTM2009-13383, MTM2010-21186-C02-01 and MTM2009-08166-E, project "Ingenio Mathematica" (i-MATH) No. CSD 2006-00032 (Consolider-Ingenio 2010), the project of the Canary Islands government SOLSUB200801000238, the ICMAT Severo Ochoa project SEV-2011-0087 and the European project IRSES-project "Geomech-246981". D. Iglesias wishes to thank MICINN for a "Ramón y Cajal" research contract. M. Vaquero wishes to thank MICINN for a FPI-PhD Position.

\section{REFERENCES}

[1] R. Abraham, J.E. Marsden, Foundations of Mechanics, Second Edition, The Benjamin-Cummings Publishing Company, New York, (1978).

[2] A. Alekseev, Y. Kosmann-Schwarzbach, Manin Pairs and moment maps, J. Differential Geom. 56 (2000) 133-165.

[3] A. Alekseev, Y. Kosmann-Schwarzbach, E. Meinrenken, Quasi-Poisson manifolds, Canad. J. Math. 54 (2002) 3-29.

[4] A. Alekseev, A. Malkin, E. Meinrenken, Lie group valued moment maps, J. Differential Geom. 48 (1998), 445-495.

[5] A. Awane: k-symplectic structures, J. Math. Phys. 33 (1992), 4046-4052.

[6] A. Awane, M. Goze, Pfaffian systems, $k$-symplectic systems. Kluwer Academic Publishers (2000).

[7] H. Bursztyn and M. Crainic, Dirac structures, momentum maps and quasi-Poisson manifolds, The breadth of symplectic and Poisson geometry 1-40, Progr. Math. 232, Birkhäuser Boston, 2005.

[8] H. Bursztyn, M. Crainic, Dirac geometry, quasi-Poisson actions, and $D / G$-valued moment maps.

[9] T. Courant, Dirac manifolds, Trans. A.M.S. 319 (1990) 631-661.

[10] C. Gunther: The polysymplectic Hamiltonian formalism in field theory and calculus of variations I: The local case, J. Differential Geom. 25 (1987), 23-53.

[11] M. Jotz, T. Ratiu: Dirac structures, nonholonomic systems and reduction. arXiv:0806.1261 v3 [math.DG]

[12] D. Li-Bland: AV-Courant Algebroids and Generalized CR Structures, Canadian Journal of Mathematics 63 (2011), 938960.

[13] P. Libermann, Ch. Marle: Symplectic geometry and analytical mechanics. Mathematics and its Applications, 35. D. Reidel Publishing Co., Dordrecht, 1987

[14] K.C.H. Mackenzie: General theory of Lie groupoids and Lie algebroids, London Mathematical Society Lecture Note Series, 213. Cambridge University Press, Cambridge, 2005.

[15] J.C. Marrero, N. Román-Roy, M. Salgado, S. Vilariño: Reduction of polysymplectic manifolds Preprint.

[16] J.E. Marsden, A. Weinstein: Reduction of symplectic manifolds with symmetry, Rep. Mathematical Phys. 5, no. 1, (1974), 121-130.

[17] R. Montgomery, J. E. Marsden, T. S. Ratiu: Gauged Lie-Poisson structures, Contemp. Math. 28 (1984) 101114, Amer. Math. Soc., Providence, RI.

[18] L. K.Norris: Symplectic geometry on $T^{*} M$ derived from $n$-symplectic geometry on $L M, J$. Geom. Phys. 13 (1994), no. 1, 5178.

[19] O. Juan-Pablo, T. Ratiu, Momentum maps and Hamiltonian reduction. Progress in Mathematics, 222. Birkhäuser Boston, Inc., Boston, MA, 2004.

[20] I.Vaisman: Lectures on the geometry of Poisson manifolds. Progress in Mathematics, 118. Birkhäuser Verlag, Basel, 1994. 
David Iglesias: Departamento de Matemática Fundamental, Facultad de Matemáticas, UniverSidAd de la Laguna, Spain

E-mail address: diglesia@ull.es

Juan Carlos Marrero: Departamento de Matemática Fundamental, Facultad de Matemáticas, Universidad De La Laguna, Spain

E-mail address: jcmarrer@ull.es

Miguel Vaquero: Instituto de Ciencias Matemáticas (CSiC-UAM- UC3M-UCM), Spain

E-mail address: miguel.vaquero@icmat.es 